University of South Florida

DIGITAL COMMONS

@ UNIVERSITY OF SOUTH FLORIDA
Digital Commons @ University of

South Florida

10-1-2007

\title{
Transit Extraboard Management-Optimum Sizing and Strategies
}

CUTR

Follow this and additional works at: https://digitalcommons.usf.edu/cutr_nctr

\section{Recommended Citation}

"Transit Extraboard Management-Optimum Sizing and Strategies," National Center for Transit Research (NCTR) Report No. CUTR-NCTR-RR-2006-01, Center for Urban Transportation Research, University of South Florida, 2007.

DOI: https://doi.org/10.5038/CUTR-NCTR-RR-2006-01

Available at: https://scholarcommons.usf.edu/cutr_nctr/168

This Technical Report is brought to you for free and open access by the National Center for Transit Research (NCTR) Archive (2000-2020) at Digital Commons @ University of South Florida. It has been accepted for inclusion in Research Reports by an authorized administrator of Digital Commons @ University of South Florida. For more information, please contact digitalcommons@usf.edu. 


\section{Transit Extraboard Management - Optimum Sizing \& Strategies}

\section{Final Report}

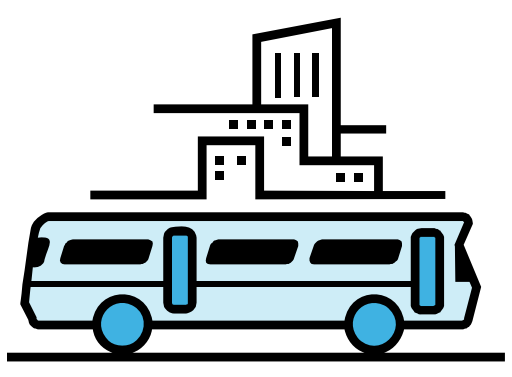

Project BD\#549-23

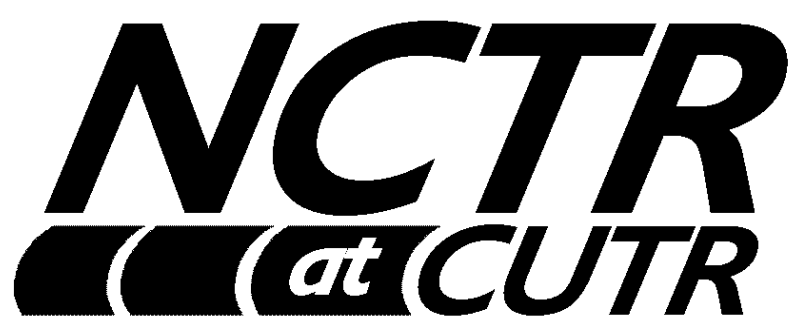

October 2007 


\title{
Transit Extraboard Management - Optimum Sizing \& Strategies Final Report
}

\section{Project \#BD549-23}

\author{
Prepared for the \\ Florida Department of Transportation \\ Research Center
}

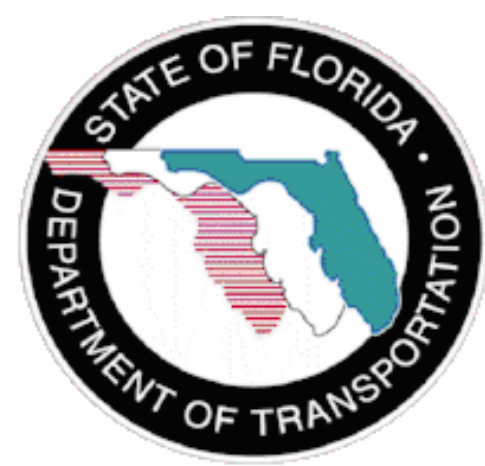

Prepared by the

National Center for Transit Research (NCTR)

Center for Urban Transportation Research University of South Florida

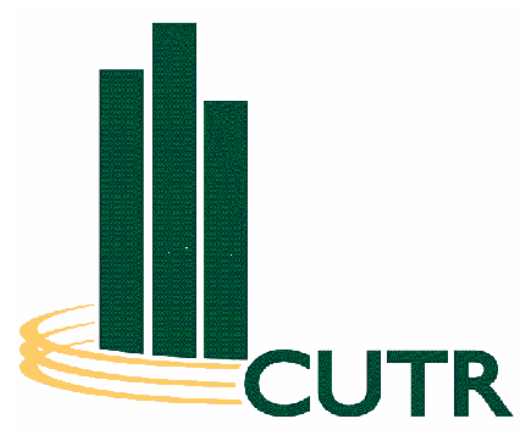

October 2007 


\section{Disclaimer}

The opinions, findings, and conclusions expressed in this publication are those of the authors and not necessarily those of the Florida Department of Transportation. 


\begin{tabular}{|c|c|c|c|c|}
\hline \multirow{3}{*}{\multicolumn{3}{|c|}{\begin{tabular}{|l|l|} 
1. Report No. & 2. Government Accession No. \\
BD549-23/ NCTR 777-07 & \\
4. Title and Subtitle \\
Transit Extraboard Management - Optimum Sizing \& \\
Strategies
\end{tabular}}} & \multicolumn{2}{|c|}{ 3. Recipient's Catalog No. } \\
\hline & & & \multicolumn{2}{|l|}{$\begin{array}{l}\text { 5. Report Date } \\
\text { October } 2007\end{array}$} \\
\hline & & & \multicolumn{2}{|c|}{ 6. Performing Organization Code } \\
\hline \multicolumn{3}{|c|}{$\begin{array}{l}\text { 7. Author(s) } \\
\text { Christopher P. DeAnnuntis and William P. Morris }\end{array}$} & \multicolumn{2}{|c|}{ 8. Performing Organization Report No. } \\
\hline \multirow{2}{*}{\multicolumn{3}{|c|}{$\begin{array}{l}\text { 9. Performing Organization Name and Address } \\
\text { National Center For Transit Research (NCTR) } \\
\text { Center for Urban Transportation Research } \\
\text { University of South Florida - CUT 100 } \\
\text { 4202 East Fowler Avenue, Tampa, FL } 33620\end{array}$}} & \multicolumn{2}{|c|}{ 10. Work Unit No. (TRAIS) } \\
\hline & & & \multicolumn{2}{|l|}{$\begin{array}{l}\text { 11. Contract or Grant No. } \\
\text { DTRS98-G-0032 } \\
\text { BD549-23 }\end{array}$} \\
\hline \multicolumn{3}{|c|}{$\begin{array}{l}\text { 12. Sponsoring Agency Name and Address } \\
\text { Office of Research and Special Programs (RSPA) } \\
\text { U.S. Department of Transportation, Washington, D.C. } \\
\text { 20590 }\end{array}$} & \multicolumn{2}{|l|}{ Final Report } \\
\hline \multicolumn{5}{|c|}{$\begin{array}{l}\text { Florida Department of Transportation } \\
605 \text { Suwannee Street, MS 26, Tallahassee, FL } 32399\end{array}$} \\
\hline \multicolumn{5}{|l|}{ 15. Supplementary Notes } \\
\hline \multicolumn{5}{|l|}{ 16. Abstract } \\
\hline \multicolumn{5}{|c|}{$\begin{array}{l}\text { The objective of this project is to summarize the process, and develop an application tool to } \\
\text { aid small to mid-size transit agencies in managing their extraboard. The application tool is } \\
\text { envisioned to work under an Excel environment to allow for easy data entry and model inputs. } \\
\text { Additionally, the project will explore practices at the State and Agency level for operator work } \\
\text { period rules and experiences. }\end{array}$} \\
\hline \multicolumn{5}{|c|}{$\begin{array}{l}\text { While transit agencies attempt to employ sufficient regular bus operators to provide scheduled } \\
\text { service, they also employ operators to cover work assignments that are temporarily unfilled } \\
\text { because of vacations, illness or absences, as well as insufficient manpower, attrition } \\
\text { (retirements, etc), or for work that is unassignable/open in the course of normal labor } \\
\text { practices. Extraboard operations may be generally defined as the process of utilizing available } \\
\text { manpower to perform work assignments in accordance with labor agreement provisions and } \\
\text { work rules to ensure the provision of scheduled transit service. The challenge is to assign } \\
\text { manpower to open scheduled work, ensuring scheduled service delivery with minimum cost. } \\
\text { Significant cost savings can result from proper extraboard management. }\end{array}$} \\
\hline \multicolumn{5}{|c|}{$\begin{array}{l}\text { Ineffective practices can increase payment of unscheduled premiums and for unproductive } \\
\text { time. Opportunities exist to improve the efficiency and effectiveness of extraboard } \\
\text { management. }\end{array}$} \\
\hline \multicolumn{2}{|c|}{$\begin{array}{l}\text { Extraboard, bus operators, scheduling, } \\
\text { operational issues, operational level model, } \\
\text { revenue hours }\end{array}$} & \multicolumn{3}{|c|}{$\begin{array}{l}\text { 18. Distribution Statement } \\
\text { Available to the public through the National } \\
\text { Technical Information Service (NTIS), } 5285 \\
\text { Port Royal Road, Springfield, VA 22161, } \\
\text { (703) 487-4650, http://www.rtis.gov/ , and } \\
\text { through the NCTR web site at } \\
\text { http://www.cutr.usf.edu/ }\end{array}$} \\
\hline \multicolumn{3}{|l|}{$\begin{array}{l}\text { 19. Security Classif. (of this report) } \\
\text { Unclassified }\end{array}$} & \begin{tabular}{|l|l|} 
Pages & 21. No. of \\
&
\end{tabular} & 22. Price \\
\hline
\end{tabular}




\section{EXECUTIVE SUMMARY}

\section{Problem and Objectives}

While transit agencies attempt to employ sufficient regular bus operators to provide scheduled service, they also employ operators to cover work assignments that are temporarily unfilled because of vacations, illness or absences, as well as insufficient manpower, attrition (retirements, etc), or for work that is unassignable/open in the course of normal labor practices. Put simply, an extraboard bus operator is another name for a back up driver. Extraboard operations may be generally defined as the process of utilizing available manpower to perform work assignments in accordance with labor agreement provisions and work rules to ensure the provision of scheduled transit service. The challenge is to assign manpower to open scheduled work, ensuring scheduled service delivery with minimum cost. Significant cost savings can result from proper extraboard management.

Four general parameters define extraboard management: available manpower; open work; labor agreement provisions, and work rules. For any given open work and given available manpower situation, there are normally several alternative decision paths. Such decisions are affected by two conditions: those established by prior actions of the transit system such as scheduling and work force planning; and those that change daily. Both have service delivery and/or financial implications. Opportunities exist to improve the efficiency and effectiveness of extraboard management.

The objective of this research is to summarize the process for and develop an application tool to aid small to mid-size transit agencies in managing their extraboard. The application tool is envisioned to work under an Excel environment to allow for easy data entry and model inputs. Additionally, the project will explore practices at the State and Agency level for operator work period rules and experiences.

\section{Findings}

Following a literature review and survey of 35 small, medium-sized and large transit agencies in the United States, NCTR researchers found the following:

- There is not a significant body of research on this topic. 
- Previous research focused on overtime pay reduction.

- Although models and methodologies had been developed in previous research, they were tested on a specific transit system and were not validated for universal use by all transit systems.

- There are differences between large, medium-sized and small transit agencies in the percentage of extraboard operators to total operators. Larger transit agencies have a much higher percentage (20\%) while small transit agencies have a much lower percentage (7\%).

- Labor contracts do impact the size, functioning and management of the extraboard.

- Although many systems responding to the survey utilize automated scheduling software, not one of them has a module for extraboard management.

- Some systems indicated that they do not currently have an extraboard due to workforce availability shortages.

- Most systems use historical data and experience to determine the size of the extraboard.

- One transit system, the Dallas Area Rapid Transit, does employ a tool to determine extraboard sizing as well as overall workforce needs.

- The DART instrument is Excel-based and consists of data that is readily available in most transit agencies, meeting the requirements of this research.

- The DART instrument was applied to a Florida transit agency and the result validates the instrument as being usable for most small and medium-sized transit agencies.

\section{Conclusions}

Consultations with operations managers in Florida revealed a number of complex circumstances regarding extraboard management, workforce recruitment and retention including:

- Absenteeism is more complex than in the past with the FMLA resulting in unpredictable and longer leaves of absence.

- Operator salaries in many Florida markets are not keeping pace with rising cost of living.

- In a hot job market, transit employees can either find higher paying jobs in the trucking industry or other sectors of the economy. 
- Recruiting shortages in many areas are driving the cost of overtime in order to meet scheduled service. Operations managers are receiving complaints from upper management about the rising costs of overtime.

- When staffing levels reach significantly low levels, overtime is forced on operators which can contribute to burnout, fatigue and increased levels of unscheduled absenteeism.

- Using actual numbers of extraboard operators for any one system is not necessarily going to reflect the true need based on total staffing requirements. This is where the DART instrument is especially helpful because it displays conclusively the levels of recruitment needed in order to maintain optimal staffing levels.

- Future research should include strategies for recruitment, salaries and incentives to achieve employee retention and absenteeism reduction. 


\section{TABLE OF CONTENTS}

TRANSIT EXTRABOARD MANAGEMENT - OPTIMUM SIZING AND STRATEGIES $\ldots . . \quad 14$

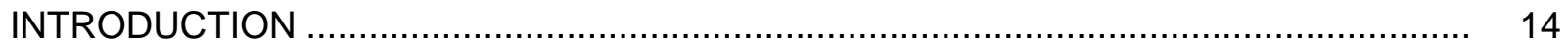

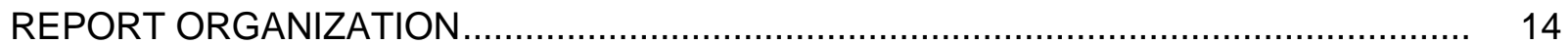

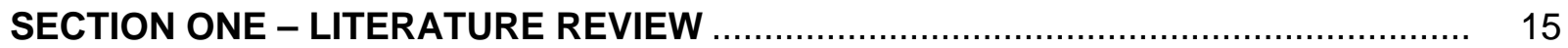

Transit Service Planning and Scheduling for Extraboard................................. 16

Operational Issues Influencing Extraboard Scheduling .................................. 18

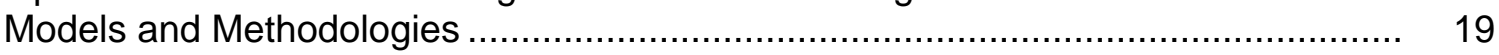

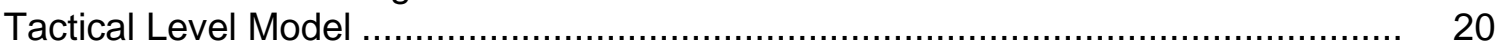

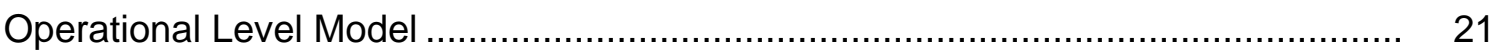

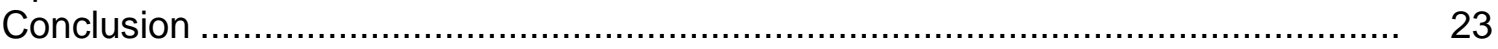

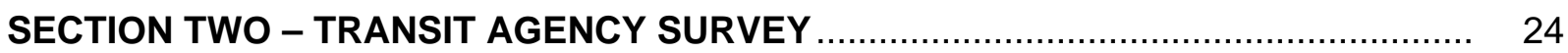

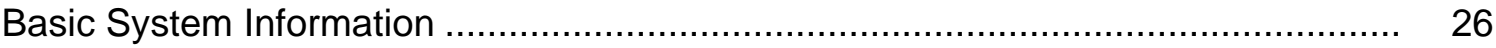

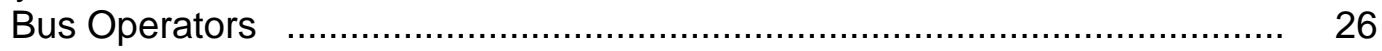

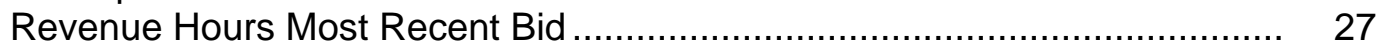

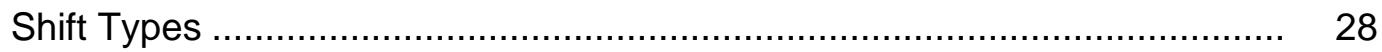

Designated Extraboard Operators...................................................... 30

Total Operators per Peak Bus ......................................................... 32

Percentage of Full Time Operators to Total Operators ............................. 32

Percentage of Part Time Operators to Total Operators ............................. 32

Ratio of Weekday Revenue Hours to Total Operators ............................... 33

Percentage of Open Work to Total Shifts ............................................... 35

Designated Extraboard Operators .................................................... 37

Percentage of Full-Time Extraboard Operators to Total Operators .............. 37

Percentage of Full Time Extraboard Operators to Full Time Operators ........ 37

Percentage of Part Time Extraboard Operators to Total Operators .............. 37

Percentage of Total Designated Extraboard (FT + PT) to Total Operators... 38

Ratio of Total Designated Extraboard Operators to Open Work ................... 38

Ratio of Total Extraboard Operators to Weekday Revenue Hours .............. $\quad 38$

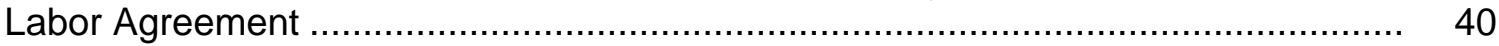

Absenteeism and Attrition ............................................................................. 41

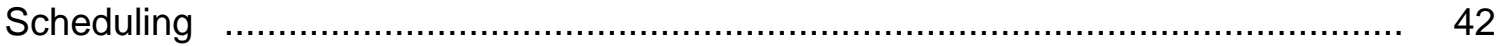

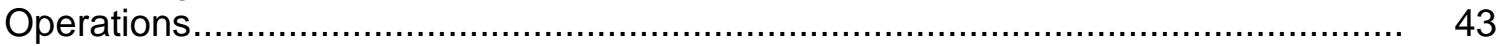

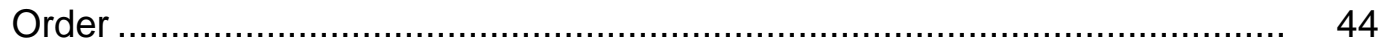

Operators Allowed to Pick .............................................................. 44

Operators Have Option to Pass ....................................................... 45

Operators Do Not Have Option to Pass ............................................... 45

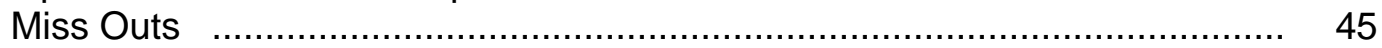

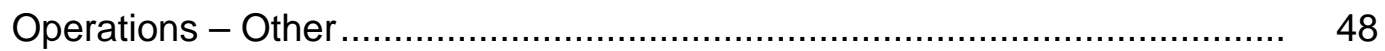

SECTION THREE - CASE STUDY AND GUIDE FOR EXTRABOARD

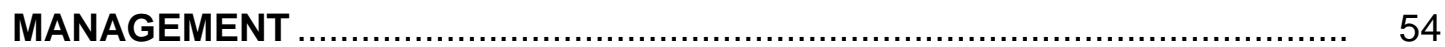

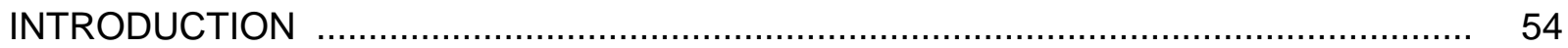

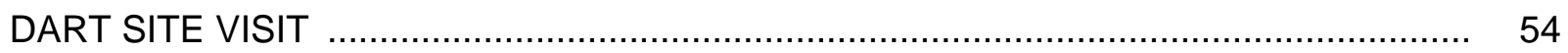

Detailed Analysis of DART Optimal Operator Report .................................. 55

Total Blocks of Work - Full Time and Part Time ................................ 55

Extraboard Requirement ............................................................... 55 


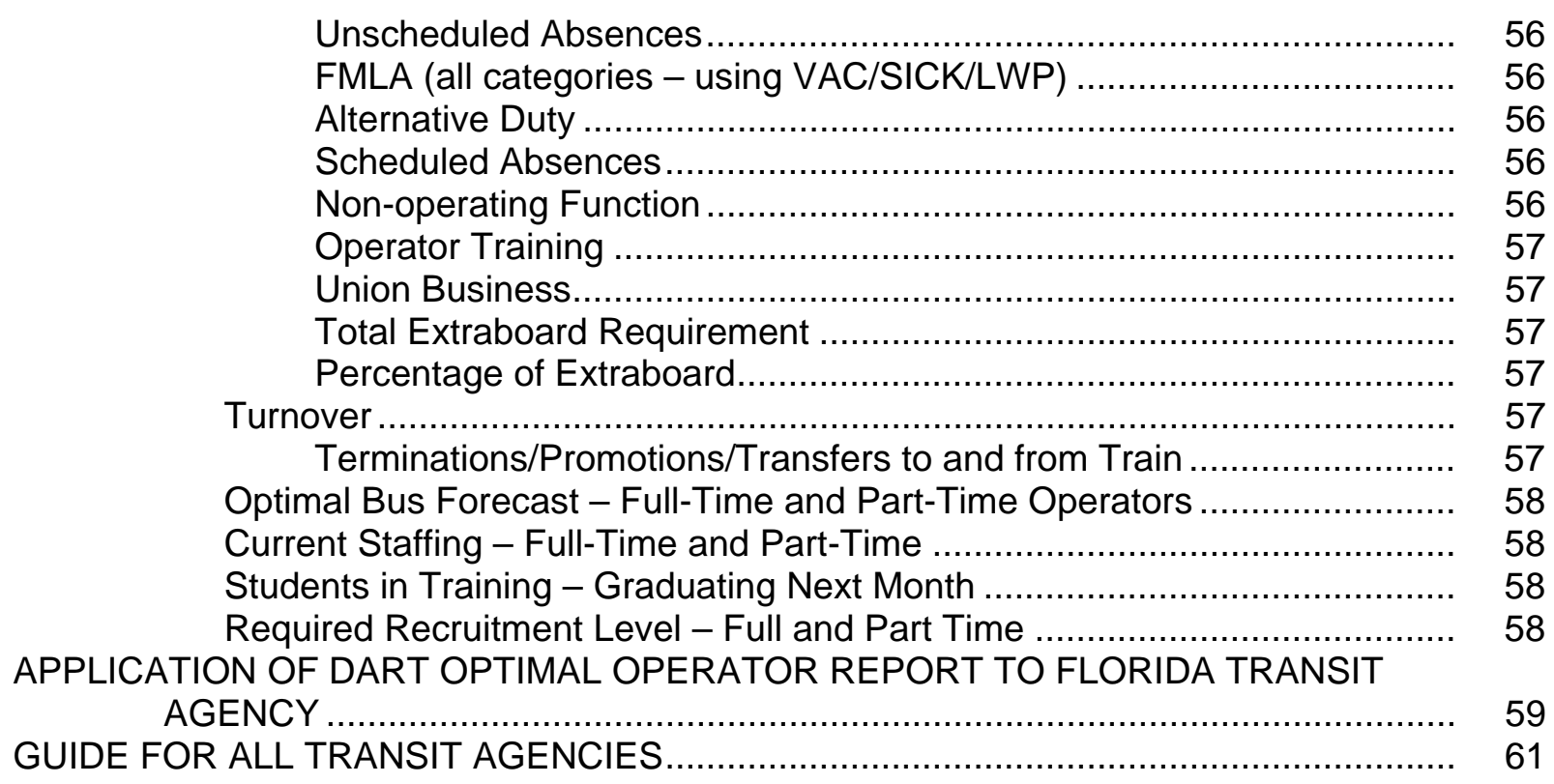

SECTION FOUR - SURVEY OF STATES - HOURS OF SERVICE (HOS) FOR DRIVERS CARRYING PASSENGERS

CFR 49, Part 395.5 - Maximum driving time for passenger-carrying vehicles............ 63

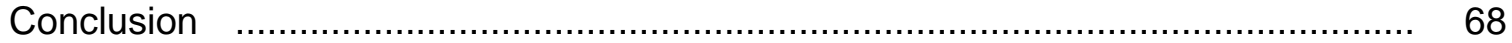

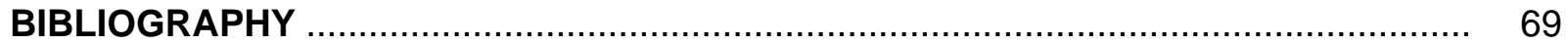

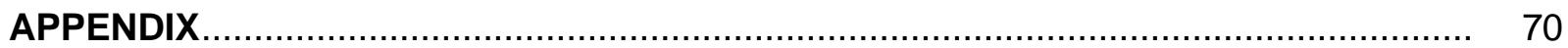

Appendix A: Transit Agency Survey Extraboard Management .............................. $\quad 70$ 


\section{LIST OF TABLES}

\section{SECTION ONE}

NONE

\section{SECTION TWO}

\section{Table 2-1}

Survey Respondents and Peak Bus Requirement FY 03-04

Table 2-2

Bus Operator Positions

Table 2-3

Revenue Hours - Weekday, Saturday and Sunday

Table 2-4

Breakdown of Shift Types - Weekday, Saturday and Sunday 29

$\underline{\text { Table 2-5 }}$

Designated Extraboard Operators

30

$\underline{\text { Table 2-6 }}$

Basic System Information Statistics - Bus Operators.

34

Table 2-7

Basic System Information Statistics - Percentage of Open Work to Total Shifts 36

Table 2-8

Basic System Information Statistics - Designated Extraboard Operators 39

Table 2-9

Labor Agreements

40

Table 2-10

Absenteeism and Attrition

Table 2-11

Scheduling

Table 2-12

Operations Practice - Assignment of Extraboard Operators

Table 2-13

DART Worksheet - Extraboard Staffing 


\section{SECTION THREE}

Table 3-1 June 2007 VOTRAN Optimal Operator Report - Three Month Rolling Average.......... 60

Table 3-2

Guide for Calculating Optimal Bus Forecast (DART)

\section{SECTION FOUR}

Table 4-1

States with Exceptions to the Federal Standard Hours of Service (HOS) for Drivers

Carrying Passengers. 


\section{LIST OF ACRONYMS}

APTA American Public Transportation Association

AASHTO American Association of State Highway and Transportation

CAT Citizens Area Transit (Las Vegas)

CDL Commercial Drivers License

COTA Central Ohio Transit Authority

CUTR Center for Urban Transportation Research

DART Dallas Area Rapid Transit

DOT Department of Transportation

FDOT Florida Department of Transportation

FMCSA Florida Motor Carrier Safety Administration

FHWA Federal Highway Administration

FMLA Family Medical Leave Act

FS Florida Statutes

FTA Federal Transit Administration

HART Hillsborough Area Regional Transit

HOS Hours of Service

JTA Jacksonville Transportation Authority

LEETRAN Lee County Transit

MBTA Massachusetts Bay Transportation Authority

NCTR National Center for Transit Research

NTD National Transit Database

SCOPT Standing Committee on Public Transportation

TRB Transportation Research Board

USDOT United States Department of Transportation

USF University of South Florida

VOTRAN Volusia County Transit

WMATA Washington Metropolitan Area Transit Authority 


\section{Transit Extraboard MANAgemEnt - OPTIMUM SizING AND STRATEgIES}

\section{INTRODUCTION}

The National Center for Transit Research commissioned this study, Transit Extraboard Management - Optimum Sizing and Strategies, to examine industry trends and identify strategies that could be used by a broad base of transit agencies to maximize extraboard sizing. The result is to be an instrument that can be universally applied to transit agency practitioners seeking to determine extraboard size based on factors other than historical trends and experience.

A literature review allowed NCTR researchers to become more familiar with topics related to extraboard management. It also provided guidance in preparation for the survey developed for the transit agencies. Researchers were interested in finding whether those issues which had been identified as having importance to managing extraboard staff, according to the literature review articles, were in fact of importance to the transit agencies that actually staff and manage extraboard operators on an operational basis. In addition, it was necessary to review and understand the empirical models and methodologies previously developed for estimating extraboards in order to assess the need for new and/or refined models that could possibly assist small, midsized and large transit agencies in managing their extraboard.

\section{REPORT ORGANIZATION}

Section One of this study is a literature review of previous studies related to extraboard management issues, strategies, models, and methodologies. Section Two details a survey of small, mid-sized and large transit agencies designed to gain information about strategies, policies, and procedures that agencies use to determine size and assignment of extraboard bus operators. Section Three is a case study conducted of the Dallas Area Rapid Transit which details an instrument that was developed by the agency to manage extraboard sizing as well as overall workforce needs for the agency. Section Three also includes applying the DART instrument to a Florida property and a guide for practitioners to use the instrument for their own agencies. Section Four is a 
survey of states requested by the Florida Department of Transportation regarding Hours of Service for Drivers Carrying Passengers. The purpose of the survey in Section Four is to identify those states that have different requirements than the minimum standard set forth by the USDOT. 


\section{Section One \\ LITERATURE REVIEW}

The results of the extraboard management literature review are presented in this section of the technical memorandum. Extensive literature searches were conducted using the TRIS literature database made available from the Transportation Research Board (TRB) and the resource library at the Center for Urban Transportation Research (CUTR). Every attempt was made to obtain and review the most current literature available. The bulk of the literature on extraboard management appeared in journals and conference proceedings between 1984 and 1990. The most current data recorded and reviewed was lecture summaries dated 2003 found on the Internet.

With the review of existing literature, researchers were looking to address two main topics to be further expanded upon during the course of this project: sizing of the extraboard and the strategies employed by transit agencies to fill the open work assignments. For sizing purposes, models would be explored to see if they assist in calculating optimum levels of extraboard needed. It was assumed that changing conditions influence the needed levels of extraboard depending on the time of day, day of week, as well as seasonal fluctuations that may result in the need for additional extraboard assignments. Strategies used by transit agencies to fill open work were assumed to consider historical leave records (reviewing scheduled as well as unscheduled leave) and agency labor rules and regulations in place.

Throughout the search for related topical literature, it was recognized that there was not a "wealth" of information regarding extraboard management. All literature collected was reviewed for relevancy to the topic and summarized. The literature reviewed addressed a variety of issues surrounding the process of extraboard management and scheduling. An emphasis was placed on prior models and methodologies developed to aid in the management and scheduling of extraboard in the transit operating environment.

The results of this literature review have been synthesized into three major categories of extraboard management issues. Those categories are: 
- service planning and scheduling for extraboard;

- operational issues influencing extraboard scheduling; and

- models and methodologies to manage extraboard size.

While there are many issues related to the effective scheduling of extraboard, the categories defined above encompass most of those issues found within the literature.

The categories are presented in the following order. The first section provides an overview of literature related to transit agency service planning and scheduling for extraboard. The second category summarizes the literature pertaining to operational issues influencing extraboard scheduling. The third category addresses models and methodologies developed to help transit operators manage extraboard size and assignments.

First, however, it is necessary to provide an operational definition of the term extraboard. According to the American Public Transportation Association (2003), the definition of extraboard is, "the roster or list of all operators who by virtue of length of service, or by choice, do not have a regularly assigned run. Part-time operators shall not be considered extraboard operators." Further, many transit agencies have defined extraboard as, "operators who have no assigned run but are used to cover runs deliberately left open by the scheduling department (extra runs), or runs that are open because of the absence of regularly assigned operators."

\section{Transit Service Planning and Scheduling for Extraboard}

The bulk of the literature on extraboard management appeared in journals and conference proceedings between 1984 and 1990. The most current data reviewed was lecture summaries dated 2003. Throughout the search for related literature, it became apparent that there was not a "wealth" of information regarding extraboard management.

According to Koutsopoulos (1990), workforce planning can be described in three levels: strategic, tactical, and operational. At the strategic level, decisions on workforce assignments are based on the size of the workforce, the hiring (staffing) levels, and the vacation schedules of the operators. Generally, the strategic level of staffing is based upon a set period of time (month or year). At the tactical level, size of the extraboard 
workforce is based on specific days of the week. Finally, at the operational level, staffing decisions are assigned based on specific times of day. Later, when developing models, Koutsopoulos focuses on the tactical and operational levels. The strategic level of staffing is what has generally occurred on a localized basis at the transit agency level. Similarly, MacDorman (1985) notes that extraboard management decisions are based on two existing conditions/activities:

- Conditions that are established by prior actions of the individual transit system, such as service planning, scheduling, and work force planning.

- Conditions that change daily and even throughout the day, such as open work.

MacDorman goes on to note that service planning, scheduling, and work force planning are all unique to the transit agency environment. Transit agencies must consider their operating environments, labor union agreements, and route structure and network issues when developing operator work assignments. Different agencies develop work assignments based on their route structure. Examples include:

- Straight run - scheduled work assignment providing the operator a full day's work on a continuous basis (8 hours typically).

- Split or swing run - peak demand conditions that often necessitate operator assignments be divided into two or more non-continuous parts to meet a full day work schedule.

- Trippers - pieces of work that cannot be matched to achieve a straight or split run.

Continuing with MacDorman, in order to meet operator need, transit agencies employ three types of operators: full-time regular, part-time, and extraboard operators. Transit agencies must determine the optimum operator staffing levels. In order to be most successful, transit agencies strive to:

- retain enough operators to ensure that no scheduled service is missed;

- avoid, when possible, paying wages when no work is performed; and

- restrict overtime pay to a level established in the transit agency's budgeting process. 
Most of the literature has identified that transit agencies have to achieve and maintain an optimum level of staffing, be it full-time, part-time, or extraboard operators. Locally, staffing must be adjusted based on service modifications, labor agreement issues and changes, and issues related to operator absence. Transit operators must internally review past staffing levels on an ongoing basis in order to meet future scheduling challenges.

\section{Operational Issues Influencing Extraboard Scheduling}

The number one operational issue that requires transit managers to staff extraboard is "open work." Open work, as noted by MacDorman, can change daily and even throughout the day, requiring transit managers to employ and retain extraboard operators. There are three main factors that result in open work:

- Unassigned service

- Non-operating assignments

- Operator absence

Often, conditions within a transit agency can result in unassigned service. Generally, unassigned service occurs when pieces of work (scheduled runs) are not selected by operators during general sign up periods, they are not part of regular operator schedules (trippers), or are related to schedule modifications and/or changes.

Within a transit agency there is often a need to staff non-operating work assignments that require operators to do things other than driving a vehicle. All transit agencies must staff training and instructional sessions, and various administrative duties. This often necessitates the need for extraboard availability.

The factor that usually dominates the need to have extraboard staffing is operator absence. All transit agencies experience operator absence based on a variety of reasons including sick leave, operators injured while on duty, personal leave, and contractual leave (vacation, holidays, etc.). The challenge created by operator absence is that some degree of absenteeism is constant and can be anticipated (such as bidding vacations in advance) while other forms of absenteeism are variable, such as sick and administrative leave. 
In addition to open work, research conducted by Long and Perry (1984) identified operational problems associated with extraboard management. Research by Long and Perry was based on surveys distributed to transit agencies within the State of California. In addition to general background agency information, the survey sought to obtain the procedures used to manage extraboard, as well as the extent of usage of the extraboard. Finally, the authors reviewed current labor agreements and policies of the transit agencies regarding extraboard. Operational problems identified by Long and Perry that were noted to have a direct relationship to extraboard management include:

- Absence incentive

- Dispatcher error

- Operator fatigue/strain

- Operator-passenger relations

- Employee morale

Based on survey results, Long and Perry found that extraboard assignments designated by historical absence patterns may result in additional absences because drivers are aware that replacement bus operators are available. Their research also found that dispatchers often assign regular operators (scheduled for day-off) open runs rather than assign open runs to the available extraboard. They found operator strain could occur when operators work long-irregular hours, causing the need for time-off and thus requiring the addition of extraboard to cover missed work. When regular operator absence occurs, it can cause service reliability issues if extraboard drivers are not comfortable and knowledgeable in the transit route system. This can detrimentally affect operator-passenger relations. Finally, Long and Perry found that employee morale may suffer if understaffing leads to the need for operators scheduled for off-days to report for duty to fill missed runs.

\section{Models and Methodologies}

Within the literature review, several models/prototypes for forecasting extraboard assignments emerged. Two models were described in Koutsopoulos' Scheduling of Extraboard Operators in Transit Systems, while a prototype automated system was developed in MacDorman's Extraboard Management: Procedures and Tools. The survey conducted by Long and Perry found that most transit agencies were using subjective and judgmental methods in determining the size of the extraboard. Most 
agencies used past experiences, as well as absence data rates in developing extraboard assignments. Their research also found some agencies using a formula developed by Peat, Marwick, Mitchell and Company which considers the optimum extraboard management to occur when the costs of non-scheduled operator overtime are balanced against the costs of guarantee pay for extraboard operators who are not utilized. And while Long and Perry found that many transit agencies prefer to understaff extraboard and rely on regular workers to receive overtime to staff needed routes, they also noted that this practice likely results in regular operators taking more unscheduled days off.

As discussed previously, Koutsopoulos developed a three-tiered approach (Strategic, Tactical, and Operational) to planning the size of the workforce including regular, parttime, and extraboard operators. Models were developed for the Tactical and Operational levels. While the objective of the tactical model was to minimize expected overtime, the objective of the operational model was to specifically address open work (noted as extra work in Koutsopoulos). In developing the two models, the author discussed and defined the problem. Then a formula (solution algorithm) was developed. Then each formula was tested within a case study using data from an actual transit agency. A brief summary of each of the models is provided below.

\section{Tactical Level Model}

This model was developed to allocate the extraboard assignments given a fixed extraboard workforce. The objective of this model was to minimize the amount of overtime for regular drivers, and instead allocate extraboard operators as needed on a daily basis. In developing this model, the following parameters were necessary for calculation:

- Total number of full time extraboard and availability for work in said time period.

- Total number of part time extraboard and availability for work in said time period.

- Total number of days off required per operator.

The tactical model was tested in a case study with data from the Massachusetts Bay Transportation Authority (MBTA). Information was obtained on the actual operator availability (full, part-time, and extraboard operators) and absences that occurred during the said survey period (data from March 1985). Policy at the MBTA required that part- 
time operators have their days off on weekends only, which was also factored into the model. The results of the model runs indicated that expected overtime levels should be 125 hours per week, whereas the actual overtime levels for the study period were 164 hours. Therefore, the model successfully reduced overtime for the period, as opposed to the methods for allocating the extraboard operators.

\section{Operational Level Model}

This model was developed to allocate the daily extraboard assignments to cover open work (extra work). The two types of open work defined by Koutsopoulos were open work known in advance (scheduled absence) and unexpected open work, usually due to operator absence (sickness). As in the tactical model, the parameters necessary for calculation include:

- Total number of full time extraboard and availability for work in said time period.

- Total number of part time extraboard and availability for work in said time period.

- Total number of days off required per operator.

In addition, it was necessary to know the work rules in place at the transit agency in order to schedule extraboard to open work whether they were straight runs, split runs, or whatever open work that needed to be filled. Additionally, the start and end times for needed runs were necessary to meet the work rules in effect at the agency. These various constraints work to complicate any models.

Once again, this model was tested in a case study with data from the MBTA. The output of this model focused on identifying report times for extraboard operators after the open runs had already been identified. Therefore, information regarding the typical start and end times were input into the model, with 15-minute intervals. The findings from the case study indicated that an average reduction of one-hour of overtime could be realized if the agency used more 2-piece assignments (two separate, non-continuous pieces of work comprising a day's work). Again, results will vary based on the individual workplace rules governing operator schedules.

MacDorman introduced an automated extraboard management system prototype in Extraboard Management: Procedures and Tools. While the report identified many elements that should be input into a computerized scheduling system, this report was 
completed in 1985, much before the technological advances that transit agencies have at their disposal in 2007. MacDorman suggested that implementing an automated system that would:

- Perform timekeeping

- Record transactions

- Maintain absence files

- Monitor costs

- Monitor service delivery

- Monitor manpower utilization

- Determine available manpower

- Determine current open work

- Maintain and manipulate operator work selection information

- Maintain and manipulate operator work assignment information

Many transit agencies now use computerized systems in existence (Trapeze, Hastus, etc.) to maintain their employee data as well as their work assignments. However, these computerized systems do not calculate extraboard needs.

\section{Conclusion}

The literature suggests that one of the primary motivations for research in the area of extraboard management is the balance of overtime pay versus regular pay to bus operators by maintaining a larger labor force. While this is a worthy reason to conduct research in this area, the fact is that there are additional factors influencing the size and utilization of an extraboard workforce. The factors that were known prior to the survey of transit agencies for this study included scheduled and unscheduled absenteeism, and work rules (labor contracts). However, there are additional factors that influence extraboard management including attrition (resignations and terminations), hold down work determined by dispatchers, and bidding processes for known open work and special assignments.

For any models that have been developed prior to technological improvements, there was at least one transit agency available for case study to test the model. However, no model developed was ever validated to be usable by any and all transit agencies including small, medium, and large transit agencies. The primary reason for the lack of 
standardization is the notation in all previous research efforts indicating that there are unique variables to all transit agencies that complicate modeling efforts. However, previous research indicates one important factor that is relevant to this research effort: most systems only use experience and prior knowledge to manage their extra-board and do not utilize rates of absenteeism or other valid data that could be used for extraboard management. This is consistent with NCTR survey research findings that indicate some systems do use established data while most others use only historical experience. 


\section{Section Two \\ Transit Agency SuRVey}

Following the literature review, CUTR conducted a survey of transit agencies across the United States. More than 400 surveys were distributed nationwide via electronic mail. A total of 35 systems responded to the survey including large transit agencies (greater than 250 buses), medium-sized transit agencies (greater than 50 and less than 250 buses), small systems (less than 50 buses), and two airport shuttle systems. The survey instrument was divided into six sections as follows:

- Basic System Information

- Labor Agreement

- Absenteeism and Attrition

- Scheduling

- Operations

- Participation in Case Study

The sections that follow will address each of these areas of the survey. Table 2-1 below shows the systems that responded to the survey and their peak bus requirement as of FY 03-04 gathered from National Transit Database (NTD) data. Peak bus requirements for Airport shuttles were not available from NTD. 
Table 2-1

Survey Respondents and Peak Bus Requirement FY 03-04

\begin{tabular}{|c|c|c|}
\hline System Type & Property & $\begin{array}{c}\text { Peak Bus } \\
\text { Requirement } \\
\text { FY 2003-04 }\end{array}$ \\
\hline $\begin{array}{l}\text { Large } \\
\text { Systems } \\
\text { >250 Buses }\end{array}$ & $\begin{array}{l}\text { Los Angeles County Metro Transp. Authority } \\
\text { Washington Metropolitan Area Transit Auth } \\
\text { Metro Transit, Minneapolis, St. Paul } \\
\text { Dallas Area Rapid Transit } \\
\text { Valley Metro, Phoenix, AZ (partial) }\end{array}$ & $\begin{array}{r}2,004 \\
1,262 \\
774 \\
440 \\
394\end{array}$ \\
\hline $\begin{array}{l}\text { Medium- } \\
\text { sized } \\
\text { Systems }>50 \\
\text { and }<250 \\
\text { Buses }\end{array}$ & $\begin{array}{l}\text { Central Ohio Transit Authority (Columbus, OH) } \\
\text { CAT, Las Vegas, NV } \\
\text { Charlotte Area Transit System (Charlotte, NC) } \\
\text { MTS San Diego } \\
\text { HART (Tampa, FL) } \\
\text { Jacksonville Transportation Authority (JTA) } \\
\text { Fairfax Connector (Fairfax, VA) } \\
\text { San Joaquin Regional Transit District (San Joaquin, CA) } \\
\text { Tempe In Motion (Tempe, AZ) } \\
\text { PRTC/First Transit (Woodbridge, VA) } \\
\text { Montebello Bus Lines (Montebello, CA) } \\
\text { Regional Transit Commission Ride (Reno) }\end{array}$ & $\begin{array}{r}247 \\
225 \\
222 \\
212 \\
154 \\
144 \\
136 \\
98 \\
78 \\
70 \\
53 \\
51\end{array}$ \\
\hline $\mid \begin{array}{l}\text { Small } \\
\text { Systems <50 } \\
\text { Buses }\end{array}$ & $\begin{array}{l}\text { VOTRAN (Daytona Beach, FL) } \\
\text { Capital Area Transit (Raliegh, NC) } \\
\text { LeeTran (Ft. Myers, FL) } \\
\text { Greater Bridgeport (Bridgeport, CT) } \\
\text { Charleston Area Regional Transit (Charleston, SC) } \\
\text { Winston Salem Transit Authority (Winston Salem, NC) } \\
\text { Escambia County Area Transit (Pensacola, FL) } \\
\text { Capital Metro, Austin, TX (partial) } \\
\text { Sarasota County Area Transit (Sarasota, FL) } \\
\text { Lakeland Area Mass Transit (Lakeland, FL) } \\
\text { Cambria County Transit Authority (Johnstown, PA) } \\
\text { Victor Valley Transit Authority (Hesperia, CA) } \\
\text { Vine \& VineGo (Napa, CA) } \\
\text { Pasco County Public Transportation (Port Richey, FL) } \\
\text { Collier County Area Transit (CAT) (Naples, FL) } \\
\text { Concord, NC }\end{array}$ & $\begin{array}{r}46 \\
46 \\
43 \\
43 \\
43 \\
37 \\
32 \\
32 \\
26 \\
25 \\
22 \\
21 \\
17 \\
14 \\
11 \\
8\end{array}$ \\
\hline Shuttles & $\begin{array}{l}\text { ShuttlePort Florida } \\
\text { AirBART }\end{array}$ & \\
\hline
\end{tabular}




\section{Basic System Information}

\section{Bus Operators}

Systems were asked to provide a breakdown of total bus operator positions, full-time and part-time. Table 2-2 below shows the breakdown of positions for each respondent.

Table 2-2

\section{Bus Operator Positions}

\begin{tabular}{|c|c|c|c|c|}
\hline System Type & Property & $\begin{array}{l}\text { Total } \\
\text { Number of } \\
\text { Bus } \\
\text { Operator } \\
\text { Positions }\end{array}$ & $\begin{array}{l}\text { Total Full } \\
\text { Time } \\
\text { Positions }\end{array}$ & $\begin{array}{l}\text { Total Part } \\
\text { Time } \\
\text { Positions }\end{array}$ \\
\hline $\begin{array}{l}\text { Large Systems } \\
>250 \text { Buses }\end{array}$ & $\begin{array}{l}\text { Los Angeles County Metro Transp. Authority } \\
\text { Washington Metropolitan Area Transit Auth } \\
\text { Metro Transit, Minneapolis, St. Paul } \\
\text { Dallas Area Rapid Transit } \\
\text { Valley Metro, Phoenix, AZ (partial) }\end{array}$ & $\begin{array}{r}3,876 \\
2,260 \\
1,436 \\
1,288 \\
682 \\
\end{array}$ & \begin{tabular}{r|}
2,905 \\
2,084 \\
1,096 \\
1,258 \\
665
\end{tabular} & \begin{tabular}{r|}
971 \\
176 \\
271 \\
30 \\
17
\end{tabular} \\
\hline $\begin{array}{l}\text { Medium-sized } \\
\text { Systems >50 } \\
\text { and <250 } \\
\text { Buses }\end{array}$ & $\begin{array}{l}\text { Central Ohio Transit Authority (Columbus, OH) } \\
\text { CAT, Las Vegas, NV } \\
\text { Charlotte Area Transit System (Charlotte, NC) } \\
\text { MTS San Diego } \\
\text { HART (Tampa, FL) } \\
\text { Jacksonville Transportation Authority (JTA) } \\
\text { Fairfax Connector (Fairfax, VA) } \\
\text { San Joaquin Regional Transit District (San Joaquin, CA) } \\
\text { Tempe In Motion (Tempe, AZ) } \\
\text { PRTC/First Transit (Woodbridge, VA) } \\
\text { Montebello Bus Lines (Montebello, CA) } \\
\text { Regional Transit Commission Ride (Reno) }\end{array}$ & \begin{tabular}{l|}
370 \\
839 \\
590 \\
290 \\
299 \\
370 \\
150 \\
170 \\
230 \\
130 \\
184 \\
197 \\
\end{tabular} & $\begin{array}{l}370 \\
832 \\
590 \\
290 \\
292 \\
363 \\
144 \\
168 \\
223 \\
125 \\
100 \\
190\end{array}$ & \begin{tabular}{r|}
0 \\
7 \\
0 \\
0 \\
7 \\
4 \\
6 \\
2 \\
7 \\
5 \\
84 \\
7
\end{tabular} \\
\hline $\begin{array}{l}\text { Small Systems } \\
<50 \text { Buses }\end{array}$ & $\begin{array}{l}\text { VOTRAN (Daytona Beach, FL) } \\
\text { Capital Area Transit (Raliegh, NC) } \\
\text { LeeTran (Ft. Myers, FL) } \\
\text { Greater Bridgeport (Bridgeport, CT) } \\
\text { Charleston Area Regional Transit (Charleston, SC) } \\
\text { Winston Salem Transit Authority (Winston Salem, NC) } \\
\text { Escambia County Area Transit (Pensacola, FL) } \\
\text { Capital Metro, Austin, TX (partial) } \\
\text { Sarasota County Area Transit (Sarasota, FL) } \\
\text { Lakeland Area Mass Transit (Lakeland, FL) } \\
\text { Cambria County Transit Authority (Johnstown, PA) } \\
\text { Victor Valley Transit Authority (Hesperia, CA) } \\
\text { Vine \& VineGo (Napa, CA) } \\
\text { Pasco County Public Transportation (Port Richey, FL) } \\
\text { Collier County Area Transit (CAT) (Naples, FL) } \\
\text { Concord, NC }\end{array}$ & $\begin{array}{r}108 \\
113 \\
132 \\
24 \\
99 \\
66 \\
65 \\
75 \\
113 \\
80 \\
46 \\
48 \\
31 \\
51 \\
35 \\
20\end{array}$ & $\begin{array}{r}107 \\
109 \\
119 \\
17 \\
99 \\
61 \\
55 \\
70 \\
113 \\
66 \\
38 \\
48 \\
31 \\
51 \\
28 \\
13\end{array}$ & $\begin{array}{r}1 \\
4 \\
13 \\
7 \\
0 \\
5 \\
10 \\
0 \\
0 \\
24 \\
8 \\
0 \\
3 \\
0 \\
10 \\
7\end{array}$ \\
\hline Shuttles & $\begin{array}{l}\text { ShuttlePort Florida } \\
\text { AirBART }\end{array}$ & $\begin{array}{r}166 \\
58\end{array}$ & $\begin{array}{r}149 \\
47\end{array}$ & $\begin{array}{l}17 \\
11\end{array}$ \\
\hline
\end{tabular}




\section{Revenue Hours Most Recent Bid}

Revenue hours are defined as the total number of hours in which a bus is in service and receiving fare-paying passengers. Revenue hours are distinguished from total hours because they do not include non-revenue time such as pre-trip inspections, "deadhead" time including travel to and from the garage, and reliefs, etc. Survey respondents were asked to provide daily revenue hours for weekdays, Saturday, and Sunday from the most recent bid. Table 2-3 below provides the breakdown for each transit agency.

Table 2-3

\section{Revenue Hours - Weekday, Saturday and Sunday}

\begin{tabular}{|c|c|c|c|c|}
\hline \multirow[b]{2}{*}{ System Type } & \multirow[b]{2}{*}{ Property } & \multicolumn{3}{|c|}{ Revenue Hours } \\
\hline & & Weekday & Saturday & Sunday \\
\hline $\begin{array}{l}\text { Large Systems } \\
>250 \text { Buses }\end{array}$ & $\begin{array}{l}\text { Los Angeles County Metro Transp. Authority } \\
\text { Washington Metropolitan Area Transit Auth } \\
\text { Metro Transit, Minneapolis, St. Paul } \\
\text { Dallas Area Rapid Transit } \\
\text { Valley Metro, Phoenix, AZ (partial) }\end{array}$ & $\begin{array}{r}22,145.0 \\
9,642.0 \\
6,026.0 \\
6,179.0 \\
2,826.4\end{array}$ & $\begin{array}{r}14,657.0 \\
4,901.0 \\
3,428.0 \\
3,629.0 \\
1,527.4\end{array}$ & $\begin{array}{r}11,590.0 \\
2,746.0 \\
2,309.0 \\
2,621.0 \\
1,100.1\end{array}$ \\
\hline $\begin{array}{l}\text { Medium-sized } \\
\text { Systems }>50 \\
\text { and }<250 \\
\text { Buses }\end{array}$ & $\begin{array}{l}\text { Central Ohio Transit Authority (Columbus, OH) } \\
\text { CAT, Las Vegas, NV } \\
\text { Charlotte Area Transit System (Charlotte, NC) } \\
\text { MTS San Diego } \\
\text { HART (Tampa, FL) } \\
\text { Jacksonville Transportation Authority (JTA) } \\
\text { Fairfax Connector (Fairfax, VA) } \\
\text { San Joaquin Regional Transit District (San Joaquin, CA) } \\
\text { Tempe In Motion (Tempe, AZ) } \\
\text { PRTC/First Transit (Woodbridge, VA) } \\
\text { Montebello Bus Lines (Montebello, CA) } \\
\text { Regional Transit Commission Ride (Reno) }\end{array}$ & $\begin{array}{r}2,104.0 \\
3,962.5 \\
2,697.5 \\
1,439.7 \\
1,709.0 \\
1,908.5 \\
887.9 \\
1,062.0 \\
1,202.0 \\
673.9 \\
800.0 \\
714.0\end{array}$ & \begin{tabular}{r|}
$1,078.0$ \\
$3,632.0$ \\
$1,456.3$ \\
$1,021.3$ \\
717.3 \\
$1,059.2$ \\
295.7 \\
465.0 \\
$1,006.5$ \\
75.9 \\
541.0 \\
627.0
\end{tabular} & $\begin{array}{r}623.0 \\
3,454.0 \\
906.2 \\
1,012.4 \\
443.0 \\
688.5 \\
219.8 \\
324.0 \\
771.4 \\
0.0 \\
429.0 \\
443.0\end{array}$ \\
\hline $\begin{array}{l}\text { Small Systems } \\
<50 \text { Buses }\end{array}$ & $\begin{array}{l}\text { VOTRAN (Daytona Beach, FL) } \\
\text { Capital Area Transit (Raliegh, NC) } \\
\text { LeeTran (Ft. Myers, FL) } \\
\text { Greater Bridgeport (Bridgeport, CT) } \\
\text { Charleston Area Regional Transit (Charleston, SC) } \\
\text { Winston Salem Transit Authority (Winston Salem, NC) } \\
\text { Escambia County Area Transit (Pensacola, FL) } \\
\text { Capital Metro, Austin, TX (partial) } \\
\text { Sarasota County Area Transit (Sarasota, FL) } \\
\text { Lakeland Area Mass Transit (Lakeland, FL) } \\
\text { Cambria County Transit Authority (Johnstown, PA) } \\
\text { Victor Valley Transit Authority (Hesperia, CA) } \\
\text { Vine \& VineGo (Napa, CA) } \\
\text { Pasco County Public Transportation (Port Richey, FL) } \\
\text { Collier County Area Transit (CAT) (Naples, FL) } \\
\text { Concord, NC }\end{array}$ & $\begin{array}{r}500.1 \\
590.5 \\
553.2 \\
105.0 \\
564.6 \\
410.0 \\
332.7 \\
423.4 \\
347.0 \\
328.0 \\
209.5 \\
256.0 \\
228.0 \\
16.0 \\
168.8 \\
91.0\end{array}$ & $\begin{array}{r}452.9 \\
414.4 \\
425.7 \\
55.0 \\
504.0 \\
219.4 \\
301.8 \\
305.4 \\
347.0 \\
254.8 \\
110.3 \\
215.0 \\
137.7 \\
0.0 \\
168.8 \\
73.0\end{array}$ & $\begin{array}{r}73.4 \\
112.5 \\
113.3 \\
30.0 \\
260.5 \\
4.3 \\
18.7 \\
182.5 \\
0.0 \\
0.0 \\
44.0 \\
0.0 \\
29.6 \\
0.0 \\
0.0 \\
0.0\end{array}$ \\
\hline Shuttles & $\begin{array}{l}\text { ShuttlePort Florida } \\
\text { AirBART }\end{array}$ & $\begin{array}{l}772.0 \\
547.0\end{array}$ & $\begin{array}{l}772.0 \\
547.0\end{array}$ & $\begin{array}{l}772.0 \\
541.0\end{array}$ \\
\hline
\end{tabular}




\section{Shift Types}

Transit agency schedulers create shifts for bus operators to perform based on local customs, work rules, and labor agreements. Shifts are broken down into different types including straights, splits, and open work. A straight shift is one wherein the entire shift is performed within a set number of hours. Typical straight shifts are divided into eighthour shifts for five day work weeks and ten-hour shifts for four day work weeks. Split shifts are those that are divided into two separate and distinct periods of the day wherein an operator will have unpaid time off between the first shift and the second shift. Split shifts usually add up to eight hours unless work rules dictate certain minimums or maximums. Open work is described as those shifts that are unassigned to an operator based on the most recent bid. Open work can also occur between bids if operators take leave based on FMLA, illness, injury, or disability. This type of open work is called a "hold down" because the shift must be held until the operator returns to work or it is deemed that the operator is permanently unable to return to work. Survey respondents were asked to provide shift types for bus operators including eight hour straights, ten hour straights, split shifts, open work, and other for weekdays, Saturday, and Sunday. Table 2-4 below provides the breakdown of shift types for each respondent. 
Table 2-4

Breakdown of Shift Types - Weekday, Saturday and Sunday

\begin{tabular}{|c|c|c|c|c|c|c|c|c|c|c|c|c|c|}
\hline \multirow[b]{2}{*}{ System Type } & \multirow[b]{2}{*}{ Property } & \multicolumn{4}{|c|}{ Weekday } & \multicolumn{4}{|c|}{ Saturday } & \multicolumn{4}{|c|}{ Sunday } \\
\hline & & $\begin{array}{l}\text { \# 8-hour } \\
\text { straights }\end{array}$ & $\begin{array}{c}\# \text { 10-hour } \\
\text { straights }\end{array}$ & $\begin{array}{c}\text { \# of } \\
\text { splits }\end{array}$ & $\begin{array}{c}\text { \# Open } \\
\text { Work }\end{array}$ & $\begin{array}{l}\text { \# 8-hour } \\
\text { straights }\end{array}$ & $\begin{array}{c}\# \text { 10-hour } \\
\text { straights }\end{array}$ & $\begin{array}{c}\text { \# of } \\
\text { splits }\end{array}$ & $\begin{array}{c}\text { \# Open } \\
\text { Work }\end{array}$ & $\begin{array}{l}\text { \# 8-hour } \\
\text { straights }\end{array}$ & $\begin{array}{c}\text { \# 10-hour } \\
\text { straights } \\
\end{array}$ & $\begin{array}{c}\text { \# of } \\
\text { splits }\end{array}$ & $\begin{array}{c}\text { \# Open } \\
\text { Work }\end{array}$ \\
\hline $\begin{array}{l}\text { Large } \\
\text { Systems } \\
>250 \text { Buses }\end{array}$ & $\begin{array}{l}\text { Los Angeles County Metro Transp. Authority } \\
\text { Washington Metropolitan Area Transit Auth } \\
\text { Metro Transit, Minneapolis, St. Paul } \\
\text { Dallas Area Rapid Transit } \\
\text { Valley Metro, Phoenix, AZ (partial) }\end{array}$ & $\begin{array}{r}1,025 \\
979 \\
208 \\
468 \\
221\end{array}$ & \begin{tabular}{r|}
100 \\
532 \\
84 \\
$\mathrm{NR}$ \\
48 \\
\end{tabular} & \begin{tabular}{r|}
740 \\
76 \\
266 \\
381 \\
173 \\
\end{tabular} & \begin{tabular}{r|}
794 \\
$\mathrm{NR}$ \\
70 \\
$\mathrm{NR}$ \\
40
\end{tabular} & $\begin{array}{r}1,145 \\
816 \\
199 \\
\mathrm{NR} \\
203 \\
\end{array}$ & \begin{tabular}{r|}
0 \\
$N R$ \\
59 \\
$N R$ \\
28 \\
\end{tabular} & \begin{tabular}{r|}
285 \\
$\mathrm{NR}$ \\
54 \\
$\mathrm{NR}$ \\
7
\end{tabular} & \begin{tabular}{r|}
480 \\
$\mathrm{NR}$ \\
94 \\
$\mathrm{NR}$ \\
0
\end{tabular} & \begin{tabular}{r|}
1,022 \\
543 \\
131 \\
$\mathrm{NR}$ \\
181
\end{tabular} & $\begin{array}{r}0 \\
\mathrm{NR} \\
42 \\
\mathrm{NR} \\
0\end{array}$ & \begin{tabular}{r|}
109 \\
$N R$ \\
34 \\
$N R$ \\
9
\end{tabular} & $\begin{array}{r}388 \\
\mathrm{NR} \\
88 \\
\mathrm{NR} \\
0\end{array}$ \\
\hline $\begin{array}{l}\text { Medium- } \\
\text { sized } \\
\text { Systems }>50 \\
\text { and <250 } \\
\text { Buses }\end{array}$ & $\begin{array}{l}\text { Central Ohio Transit Authority (Columbus, OH) } \\
\text { CAT, Las Vegas, NV } \\
\text { Charlotte Area Transit System (Charlotte, NC) } \\
\text { MTS San Diego } \\
\text { HART (Tampa, FL) } \\
\text { Jacksonville Transportation Authority (JTA) } \\
\text { Fairfax Connector (Fairfax, VA) } \\
\text { San Joaquin Regional Transit District (San Joaquin, CA) } \\
\text { Tempe In Motion (Tempe, AZ) } \\
\text { PRTC/First Transit (Woodbridge, VA) } \\
\text { Montebello Bus Lines (Montebello, CA) } \\
\text { Regional Transit Commission Ride (Reno) }\end{array}$ & \begin{tabular}{r|}
163 \\
233 \\
135 \\
191 \\
108 \\
169 \\
44 \\
15 \\
49 \\
40 \\
84 \\
54
\end{tabular} & \begin{tabular}{r|r}
0 \\
181 \\
76 \\
6 \\
18 \\
20 \\
13 \\
13 \\
31 \\
0 \\
9 \\
25 \\
\end{tabular} & \begin{tabular}{r|}
124 \\
47 \\
164 \\
84 \\
91 \\
63 \\
33 \\
73 \\
50 \\
66 \\
8 \\
$N R$
\end{tabular} & $\begin{array}{r}17 \\
24 \\
69 \\
24 \\
12 \\
0 \\
9 \\
0 \\
25 \\
2 \\
5 \\
12\end{array}$ & $\begin{array}{r}91 \\
208 \\
126 \\
119 \\
37 \\
82 \\
35 \\
7 \\
37 \\
9 \\
34 \\
50\end{array}$ & $\begin{array}{r}0 \\
189 \\
40 \\
0 \\
21 \\
16 \\
21 \\
6 \\
67 \\
0 \\
1 \\
19\end{array}$ & $\begin{array}{r}38 \\
27 \\
26 \\
32 \\
27 \\
24 \\
14 \\
16 \\
2 \\
0 \\
2\end{array}$ & $\begin{array}{r}8 \\
17 \\
29 \\
20 \\
6 \\
0 \\
0 \\
1 \\
16 \\
0 \\
9 \\
11\end{array}$ & $\begin{array}{r}24 \\
196 \\
85 \\
114 \\
23 \\
42 \\
27 \\
4 \\
49 \\
0 \\
27 \\
34\end{array}$ & $\begin{array}{r}0 \\
185 \\
25 \\
0 \\
12 \\
14 \\
22 \\
5 \\
41 \\
0 \\
1 \\
14\end{array}$ & \begin{tabular}{r|}
10 \\
24 \\
15 \\
33 \\
21 \\
18 \\
5 \\
12 \\
0 \\
0 \\
2
\end{tabular} & $\begin{array}{r}70 \\
14 \\
27 \\
4 \\
6 \\
0 \\
0 \\
0 \\
16 \\
0 \\
9 \\
6\end{array}$ \\
\hline $\begin{array}{l}\text { Small } \\
\text { Systems < } 50 \\
\text { Buses }\end{array}$ & $\begin{array}{l}\text { VOTRAN (Daytona Beach, FL) } \\
\text { Capital Area Transit (Raliegh, NC) } \\
\text { LeeTran (Ft. Myers, FL) } \\
\text { Greater Bridgeport (Bridgeport, CT) } \\
\text { Charleston Area Regional Transit (Charleston, SC) } \\
\text { Winston Salem Transit Authority (Winston Salem, NC) } \\
\text { Escambia County Area Transit (Pensacola, FL) } \\
\text { Capital Metro, Austin, TX (partial) } \\
\text { Sarasota County Area Transit (Sarasota, FL) } \\
\text { Lakeland Area Mass Transit (Lakeland, FL) } \\
\text { Cambria County Transit Authority (Johnstown, PA) } \\
\text { Victor Valley Transit Authority (Hesperia, CA) } \\
\text { Vine \& VineGo (Napa, CA) } \\
\text { Pasco County Public Transportation (Port Richey, FL) } \\
\text { Collier County Area Transit (CAT) (Naples, FL) } \\
\text { Concord, NC }\end{array}$ & \begin{tabular}{r|}
36 \\
28 \\
262 \\
10 \\
52 \\
22 \\
13 \\
23 \\
43 \\
$N R$ \\
19 \\
32 \\
20 \\
2 \\
14 \\
7 \\
\end{tabular} & \begin{tabular}{r|r}
6 \\
9 \\
105 \\
0 \\
0 \\
6 \\
4 \\
21 \\
0 \\
$\mathrm{NR}$ \\
0 \\
0 \\
0 \\
$\mathrm{NR}$ \\
0 \\
1
\end{tabular} & $\begin{array}{r}20 \\
34 \\
1 \\
8 \\
19 \\
27 \\
24 \\
26 \\
11 \\
\mathrm{NR} \\
5 \\
0 \\
5 \\
\mathrm{NR} \\
9 \\
0\end{array}$ & $\begin{array}{r}4 \\
0 \\
1 \\
5 \\
0 \\
0 \\
0 \\
N R \\
1 \\
0 \\
6 \\
N R \\
5 \\
4\end{array}$ & $\begin{array}{r}31 \\
24 \\
40 \\
6 \\
46 \\
29 \\
14 \\
26 \\
43 \\
\mathrm{NR} \\
13 \\
32 \\
20 \\
\mathrm{NR} \\
10 \\
5\end{array}$ & $\begin{array}{r}4 \\
19 \\
16 \\
0 \\
0 \\
0 \\
0 \\
10 \\
0 \\
\mathrm{NR} \\
0 \\
0 \\
0 \\
\mathrm{NR} \\
0 \\
0\end{array}$ & $\begin{array}{r}15 \\
7 \\
1 \\
0 \\
17 \\
5 \\
16 \\
1 \\
11 \\
\mathrm{NR} \\
3 \\
0 \\
2 \\
\mathrm{NR} \\
7 \\
0\end{array}$ & $\begin{array}{r}4 \\
0 \\
1 \\
1 \\
2 \\
5 \\
0 \\
0 \\
0 \\
\mathrm{NR} \\
0 \\
0 \\
5 \\
\mathrm{NR} \\
8 \\
4\end{array}$ & $\begin{array}{r}4 \\
10 \\
12 \\
4 \\
23 \\
0 \\
2 \\
14 \\
0 \\
\mathrm{NR} \\
6 \\
0 \\
3 \\
\mathrm{NR} \\
0 \\
0\end{array}$ & $\begin{array}{r}0 \\
0 \\
2 \\
0 \\
0 \\
0 \\
0 \\
4 \\
0 \\
\mathrm{NR} \\
0 \\
0 \\
0 \\
\mathrm{NR} \\
0 \\
0\end{array}$ & $\begin{array}{r}3 \\
4 \\
0 \\
0 \\
9 \\
1 \\
1 \\
4 \\
0 \\
\mathrm{NR} \\
0 \\
0 \\
0 \\
\mathrm{NR} \\
0 \\
0\end{array}$ & $\begin{array}{r}2 \\
0 \\
1 \\
1 \\
3 \\
0 \\
0 \\
0 \\
0 \\
\mathrm{NR} \\
0 \\
0 \\
2 \\
\mathrm{NR} \\
0 \\
0\end{array}$ \\
\hline Shuttles & $\begin{array}{l}\text { ShuttlePort Florida } \\
\text { AirBART }\end{array}$ & $\begin{array}{r}259 \\
31\end{array}$ & $\begin{array}{r}166 \\
6\end{array}$ & $\begin{array}{l}0 \\
1\end{array}$ & $\begin{array}{r}83 \\
0\end{array}$ & $\begin{array}{r}259 \\
31\end{array}$ & $\begin{array}{r}166 \\
6\end{array}$ & $\begin{array}{l}0 \\
1\end{array}$ & $\begin{array}{r}83 \\
0\end{array}$ & $\begin{array}{r}259 \\
36\end{array}$ & $\begin{array}{r}166 \\
2\end{array}$ & $\begin{array}{l}0 \\
1\end{array}$ & 83 \\
\hline
\end{tabular}

NR $=$ No Response 


\section{Designated Extraboard Operators}

Respondents were asked to provide a breakdown of the size of the dedicated extraboard with full time and part time operators. The responses revealed that a majority of designated extraboard operators are full time. Only three systems that responded to the survey, Montebello, ECAT, and Collier Area Transit, use a significant number of part time operators for the extraboard. Table 2-5 below shows the breakdown for each survey respondent.

Table 2-5

Designated Extraboard Operators

\begin{tabular}{|c|c|c|c|c|}
\hline System Type & Property & $\begin{array}{l}\text { Designated } \\
\text { Full Time } \\
\text { Extraboard } \\
\end{array}$ & $\begin{array}{l}\text { Designated } \\
\text { Part Time } \\
\text { Extraboard }\end{array}$ & $\begin{array}{c}\text { Total } \\
\text { Designated } \\
\text { Extraboard } \\
\end{array}$ \\
\hline $\begin{array}{l}\text { Large } \\
\text { Systems } \\
>250 \text { Buses }\end{array}$ & \begin{tabular}{|l} 
Los Angeles County Metro Transp. Authority \\
Washington Metropolitan Area Transit Auth \\
Metro Transit, Minneapolis, St. Paul \\
Dallas Area Rapid Transit \\
Valley Metro, Phoenix, AZ (partial)
\end{tabular} & $\begin{array}{r}1,000 \\
316 \\
280 \\
239 \\
136\end{array}$ & $\begin{array}{l}0 \\
0 \\
0 \\
0 \\
0\end{array}$ & \begin{tabular}{r|}
1,000 \\
316 \\
280 \\
239 \\
136 \\
\end{tabular} \\
\hline $\begin{array}{l}\text { Medium- } \\
\text { sized } \\
\text { Systems >50 } \\
\text { and <250 } \\
\text { Buses }\end{array}$ & $\begin{array}{l}\text { Central Ohio Transit Authority (Columbus, OH) } \\
\text { CAT, Las Vegas, NV } \\
\text { Charlotte Area Transit System (Charlotte, NC) } \\
\text { MTS San Diego } \\
\text { HART (Tampa, FL) } \\
\text { Jacksonville Transportation Authority (JTA) } \\
\text { Fairfax Connector (Fairfax, VA) } \\
\text { San Joaquin Regional Transit District (San Joaquin, CA) } \\
\text { Tempe In Motion (Tempe, AZ) } \\
\text { PRTC/First Transit (Woodbridge, VA) } \\
\text { Montebello Bus Lines (Montebello, CA) } \\
\text { Regional Transit Commission Ride (Reno) }\end{array}$ & \begin{tabular}{r|r}
72 \\
82 \\
113 \\
9 \\
42 \\
46 \\
14 \\
31 \\
14 \\
18 \\
0 \\
39
\end{tabular} & $\begin{array}{r}0 \\
5 \\
0 \\
0 \\
0 \\
0 \\
0 \\
0 \\
3 \\
6 \\
24 \\
7 \\
\end{array}$ & $\begin{array}{r}72 \\
87 \\
113 \\
9 \\
42 \\
46 \\
14 \\
31 \\
17 \\
24 \\
24 \\
46\end{array}$ \\
\hline $\begin{array}{l}\text { Small } \\
\text { Systems }<50 \\
\text { Buses }\end{array}$ & $\begin{array}{l}\text { VOTRAN (Daytona Beach, FL) } \\
\text { Capital Area Transit (Raliegh, NC) } \\
\text { LeeTran (Ft. Myers, FL) } \\
\text { Greater Bridgeport (Bridgeport, CT) } \\
\text { Charleston Area Regional Transit (Charleston, SC) } \\
\text { Winston Salem Transit Authority (Winston Salem, NC) } \\
\text { Escambia County Area Transit (Pensacola, FL) } \\
\text { Capital Metro, Austin, TX (partial) } \\
\text { Sarasota County Area Transit (Sarasota, FL) } \\
\text { Lakeland Area Mass Transit (Lakeland, FL) } \\
\text { Cambria County Transit Authority (Johnstown, PA) } \\
\text { Victor Valley Transit Authority (Hesperia, CA) } \\
\text { Vine \& VineGo (Napa, CA) } \\
\text { Pasco County Public Transportation (Port Richey, FL) } \\
\text { Collier County Area Transit (CAT) (Naples, FL) } \\
\text { Concord, NC }\end{array}$ & \begin{tabular}{r|r}
20 \\
4 \\
8 \\
0 \\
7 \\
3 \\
5 \\
5 \\
14 \\
$N R$ \\
5 \\
0 \\
1 \\
2 \\
0 \\
2
\end{tabular} & \begin{tabular}{r|r}
1 \\
0 \\
0 \\
0 \\
0 \\
5 \\
10 \\
0 \\
0 \\
$\mathrm{NR}$ \\
8 \\
0 \\
0 \\
0 \\
10 \\
0
\end{tabular} & \begin{tabular}{r|r}
21 \\
4 \\
8 \\
0 \\
7 \\
8 \\
15 \\
5 \\
14 \\
$N R$ \\
13 \\
0 \\
1 \\
2 \\
10 \\
2
\end{tabular} \\
\hline Shuttles & $\begin{array}{l}\text { ShuttlePort Florida } \\
\text { AirBART }\end{array}$ & $\begin{array}{l}5 \\
0\end{array}$ & $\begin{array}{l}0 \\
9\end{array}$ & \\
\hline
\end{tabular}

Statistics - Basic System Information 
From the basic system information, CUTR was able to develop a series of statistical calculations for each of the systems that supplied data, as follows:

- Total Operators per Peak Bus Requirement

- Percentage of Total Full Time Operators to Total Operators

- Percentage of Total Part Time Operators to Total Operators

- Ratio of Weekday Revenue Hours to Total Operators

- Percentage of Open Work to Total Shifts

- Percentage of Full Time Extraboard Operators to Total Operators

- Percentage of Full-Time Extraboard Operators to Total Full Time Operators

- Percentage of Part-Time Extraboard Operators to Total Operators

- Percentage of Total Designated Extraboard Operators to Total Operators

- Percentage of Total Designated Extraboard Operators to Total Full Time Operators

- Ratio of Total Extraboard Operators to Open Work

- Ratio of Total Extraboard Operators to Weekday Revenue Hours

Since previous literature did not show that surveys were performed and statistical analyses were reported, NCTR researchers took the opportunity to conduct this examination and explore whether this type of data could be useful and beneficial for practitioners and future researchers. The narrative below, at a minimum, provides benefits to those agencies participating in the survey, as follows:

- Statistical data displays consistencies or inconsistencies in comparisons between transit agencies and between large, medium-size, and small properties.

- Statistical data enables individual systems to see how their own data compares to the mean for the group.

- Statistical data enables practitioners to compare their own data to those reported in this study.

- The possibility that examining data of this nature could impact individual system performance, effectiveness, and/or efficiency. 
Total Operators per Peak Bus

When examining this data set, it is noted that peak bus requirement refers to weekdays only whereas total operators are those required to perform weekday, Saturday, and Sunday service. Each system will have different levels of service that determine the total number of operators required. With this caveat, the data suggests that when comparing mean values, the ratio of total operators to peak bus requirement is between 2.0 and 2.5 total operators per peak bus. For large systems, the mean is 2.05 operators per peak bus with a maximum of 2.93 (DART); for medium-size systems the mean value is 2.40 with a maximum of 3.73 (Las Vegas); and for small systems the mean value is 2.50 with a maximum of 4.35 (Sarasota). Peak bus requirements were not available from NTD for shuttle services; therefore there is no data set for this statistic. Because of differences in levels of service between weekdays, Saturday, and Sunday, this statistical data set is not used to determine potential impacts on system performance.

\section{Percentage of Full Time Operators to Total Operators}

When comparing mean values for this data set, the range of full time operators to total operators is from 88 percent to 95 percent of total operators for large, medium-size, and small transit agencies. For large transit agencies, the mean value is 88 percent with a maximum of 98 percent (DART); for medium-size properties the mean value is 95 percent with a maximum of 100 percent (Columbus, Charlotte, and San Diego); and for small transit agencies the mean value is 90 percent with a maximum of 100 percent (Charleston, Sarasota, Hesperia, Napa, and Pasco). For shuttles, the mean value is 85 percent with a maximum of 90 percent (Shuttleport Florida).

\section{Percentage of Part Time Operators to Total Operators}

When comparing mean values for this data set, the range of part time operators to total operators is 5.5 percent to 11.7 percent. For large transit agencies, the mean value is 11.3 percent with a maximum of 25 percent (Los Angeles). For medium size transit agencies the mean value is 5.5 percent with a maximum of 45.7 percent (Montebello), and for small transit agencies the mean value is 11.7 percent with a maximum of 35 percent (Concord). For shuttles, the mean value is 14.6 percent with a maximum of 19 percent (AirBART). 
The breakdown of full time and part time operators can play an important role in performance and efficiency for transit agencies because scheduled service may require a higher percentage of part time operators (e.g., more service in peak periods, less service in base periods) but labor agreements can hold down, limit, or prohibit part time operators. When this happens, the transit agency may be subject to minimum pay guarantees for full-time operators when the pay hours are actually commensurate with a part time position. Correspondingly, labor agreements can also limit and/or prohibit the breakdown of the full time vs. part time composition of the extraboard.

\section{Ratio of Weekday Revenue Hours to Total Operators}

When comparing the mean values for this data set, the range of the ratio of weekday revenue hours to total operators is 4.62 to 5.11 for small, medium-size, and large transit agencies. For large transit agencies, the mean value is 4.62 revenue hours per operator with a maximum of 5.71 revenue hours per operator (Los Angeles). For medium size transit agencies, the mean value is 5.11 revenue hours per operator with a maximum of 5.72 (Tampa). For small transit agencies, the mean value is 4.70 revenue hours per operator with a maximum value of 7.35 (Napa). For shuttles, the mean value is 7.04 with a maximum of 9.43 (AirBART). Of all the statistical data sets examined, this set has a high degree of correlation between transit systems of all sizes (except shuttles).

A higher ratio of weekday revenue hours to total bus operators means that the transit agency is maximizing its labor force. When the ratio is lower, it means the transit system may be employing too many operators to meet necessary scheduled service. Table 2-6 below displays the statistics for bus operators for large, medium-size, small transit agencies, and shuttles. 
Table 2-6

Basic System Information Statistics - Bus Operators

\begin{tabular}{|c|c|c|c|c|c|c|c|c|c|c|}
\hline System Type & Property & $\begin{array}{c}\text { Peak Bus } \\
\text { Requirement } \\
\text { FY 2003-04 }\end{array}$ & $\begin{array}{l}\text { Total Bus } \\
\text { Operator } \\
\text { Positions }\end{array}$ & $\begin{array}{c}\text { Total } \\
\text { Operators per } \\
\text { Peak Bus }\end{array}$ & $\begin{array}{c}\text { Total Full } \\
\text { Time } \\
\text { Positions }\end{array}$ & \begin{tabular}{|c|} 
Percentage \\
of Total Full \\
Time \\
Operators to \\
Total \\
Operators
\end{tabular} & $\begin{array}{c}\text { Total Part } \\
\text { Time } \\
\text { Operators }\end{array}$ & $\begin{array}{c}\text { Percentage } \\
\text { of Total Part } \\
\text { Time } \\
\text { Operators } \\
\text { to Total } \\
\text { Operators }\end{array}$ & $\begin{array}{c}\text { Weekday } \\
\text { Revenue } \\
\text { Hours }\end{array}$ & $\begin{array}{c}\text { Ratio of } \\
\text { Weekday } \\
\text { Revenue } \\
\text { Hours to } \\
\text { Total } \\
\text { Operators }\end{array}$ \\
\hline \multirow{6}{*}{$\begin{array}{l}\text { Large } \\
\text { Systems } \\
>250 \text { Buses }\end{array}$} & Los Angeles County Metro Transp. Authority & 2,004 & 3,876 & 1.93 & 2,905 & $74.9 \%$ & 971 & $25.1 \%$ & 22,145 & 5.71 \\
\hline & Washington Metropolitan Area Transit Auth & 1,262 & 2,260 & 1.79 & 2,084 & $92.2 \%$ & 176 & $7.8 \%$ & 9,642 & 4.27 \\
\hline & Metro Transit, Minneapolis, St. Paul & 774 & 1,436 & 1.86 & 1,096 & $76.3 \%$ & 271 & $18.9 \%$ & 6,026 & 4.20 \\
\hline & Dallas Area Rapid Transit & 440 & 1,288 & 2.93 & 1,258 & $97.7 \%$ & 30 & $2.3 \%$ & 6,179 & 4.80 \\
\hline & Valley Metro, Phoenix, AZ (partial) & 394 & & 1.73 & 665 & $97.5 \%$ & 17 & $2.5 \%$ & 2,826 & 4.14 \\
\hline & MEAN & 975 & 1,908 & 2.05 & 1,602 & $87.7 \%$ & 293 & $11.3 \%$ & 9,364 & 4.62 \\
\hline \multirow{13}{*}{$\begin{array}{l}\text { Medium- } \\
\text { sized } \\
\text { Systems >50 } \\
\text { and <250 } \\
\text { Buses }\end{array}$} & Central Ohio Transit Authority (Columbus, $\mathrm{OH}$ ) & 247 & 370 & 1.50 & 370 & $100.0 \%$ & 0 & $0.0 \%$ & 2,104 & 5.69 \\
\hline & CAT, Las Vegas, NV & 225 & 839 & 3.73 & 832 & $99.2 \%$ & 7 & $0.8 \%$ & 3,963 & 4.72 \\
\hline & Charlotte Area Transit System (Charlotte, NC) & 222 & 590 & 2.66 & 590 & $100.0 \%$ & 0 & $0.0 \%$ & 2,698 & 4.57 \\
\hline & MTS San Diego & 212 & 290 & 1.37 & 290 & $100.0 \%$ & 0 & $0.0 \%$ & 1,440 & 4.96 \\
\hline & HART (Tampa, FL) & 154 & 299 & 1.94 & 292 & $97.7 \%$ & 7 & $2.3 \%$ & 1,709 & 5.72 \\
\hline & Jacksonville Transportation Authority (JTA) & 144 & 370 & 2.57 & 363 & $98.1 \%$ & 4 & $1.1 \%$ & 1,908 & 5.16 \\
\hline & Fairfax Connector (Fairfax, VA) & 136 & 150 & 1.10 & 144 & $96.0 \%$ & 6 & $4.0 \%$ & 888 & 5.92 \\
\hline & San Joaquin Regional Transit District (San Joaquin, CA) & 98 & 170 & 1.73 & 168 & $98.8 \%$ & 2 & $1.2 \%$ & 1,062 & 6.25 \\
\hline & Tempe In Motion (Tempe, AZ) & 78 & 230 & 2.95 & 223 & $97.0 \%$ & 7 & $3.0 \%$ & 1,202 & 5.23 \\
\hline & PRTC/First Transit (Woodbridge, VA) & 70 & 130 & 1.86 & 125 & $96.2 \%$ & 5 & $3.8 \%$ & 674 & 5.18 \\
\hline & Montebello Bus Lines (Montebello, CA) & 53 & 184 & 3.47 & 100 & $54.3 \%$ & 84 & $45.7 \%$ & 800 & 4.35 \\
\hline & Regional Transit Commission Ride (Reno) & 51 & 197 & 3.86 & 190 & $96.4 \%$ & & $3.6 \%$ & 714 & 3.62 \\
\hline & MEAN & 141 & 318 & 2.40 & 307 & $94.5 \%$ & 11 & $5.5 \%$ & 1,597 & 5.11 \\
\hline \multirow{17}{*}{$\mid \begin{array}{l}\text { Small } \\
\text { Systems <50 } \\
\text { Buses }\end{array}$} & VOTRAN (Daytona Beach, FL) & 46 & 108 & 2.35 & 107 & $99.1 \%$ & 1 & $0.9 \%$ & 500 & 4.63 \\
\hline & Capital Area Transit (Raliegh, NC) & 46 & 113 & 2.46 & 109 & $96.5 \%$ & 4 & $3.5 \%$ & 591 & 5.23 \\
\hline & LeeTran (Ft. Myers, FL) & 43 & 132 & 3.07 & 119 & $90.2 \%$ & 13 & $9.8 \%$ & 553 & 4.19 \\
\hline & Greater Bridgeport (Bridgeport, CT) & 43 & 24 & 0.56 & 17 & $70.8 \%$ & 7 & $29.2 \%$ & 105 & 4.38 \\
\hline & Charleston Area Regional Transit (Charleston, SC) & 43 & 99 & 2.30 & 99 & $100.0 \%$ & 0 & $0.0 \%$ & 565 & 5.70 \\
\hline & Winston Salem Transit Authority (Winston Salem, NC) & 37 & 66 & 1.78 & 61 & $92.4 \%$ & 5 & $7.6 \%$ & 410 & 6.21 \\
\hline & Escambia County Area Transit (Pensacola, FL) & 32 & 65 & 2.03 & 55 & $84.6 \%$ & 10 & $15.4 \%$ & 333 & 5.12 \\
\hline & Capital Metro, Austin, TX (partial) & 32 & 75 & 2.34 & 70 & $93.3 \%$ & 0 & $0.0 \%$ & 423 & 5.65 \\
\hline & Sarasota County Area Transit (Sarasota, FL) & 26 & 113 & 4.35 & 113 & $100.0 \%$ & 0 & $0.0 \%$ & 347 & 3.07 \\
\hline & Lakeland Area Mass Transit (Lakeland, FL) & 25 & 80 & 3.20 & 66 & $82.5 \%$ & 24 & $30.0 \%$ & 328 & 4.10 \\
\hline & Cambria County Transit Authority (Johnstown, PA) & 22 & 46 & 2.09 & 38 & $82.6 \%$ & 8 & $17.4 \%$ & 210 & 4.55 \\
\hline & Victor Valley Transit Authority (Hesperia, CA) & 21 & 48 & 2.29 & 48 & $100.0 \%$ & 0 & $0.0 \%$ & 256 & 5.33 \\
\hline & Vine \& VineGo (Napa, CA) & 17 & 31 & 1.82 & 31 & $100.0 \%$ & 3 & $9.7 \%$ & 228 & 7.35 \\
\hline & Pasco County Public Transportation (Port Richey, FL) & 14 & 51 & 3.64 & 51 & $100.0 \%$ & 0 & $0.0 \%$ & 16 & 0.31 \\
\hline & Collier County Area Transit (CAT) (Naples, FL) & 11 & 35 & 3.18 & 28 & $80.0 \%$ & 10 & $28.6 \%$ & 169 & 4.82 \\
\hline & Concord, NC & & 20 & 2.50 & 13 & $65.0 \%$ & 7 & $35.0 \%$ & 91 & 4.55 \\
\hline & MEAN & 29 & 69 & 2.50 & 64 & $89.8 \%$ & 6 & $11.7 \%$ & 320 & 4.70 \\
\hline \multirow{3}{*}{ Shuttles } & ShuttlePort Florida & $\mathrm{N} / \mathrm{A}$ & 166 & N/A & 149 & $89.8 \%$ & 17 & $10.2 \%$ & 772 & 4.65 \\
\hline & Air & $\mathrm{N} / \mathrm{A}$ & 58 & N/A & 47 & $81.0 \%$ & 11 & $19.0 \%$ & 547 & 9.43 \\
\hline & MEAN & N/A & 112 & N/A & 98 & $85.4 \%$ & 14 & $14.6 \%$ & 660 & 7.04 \\
\hline
\end{tabular}




\section{Percentage of Open Work to Total Shifts}

As defined in the "Shift Types" section above, open work is described as those shifts that are unassigned to an operator based on the most recent bid. Open work can also occur between bids if operators take leave based on FMLA, illness, injury, or disability. This type of open work is called a "hold down" because the shift must be held until the operator returns to work or it is deemed that the operator is permanently unable to return to work.

To report this data set, total shifts had to be calculated for weekdays in order to determine the percentage of open work to total shifts. This data set is significant because open work is most likely to be performed by the extraboard and is an important factor for transit agencies to consider when determining the size of the board.

When comparing the mean values between large, medium-size, and small transit agencies, the range of percentages is from 6.2 percent for small transit agencies to 9.9 percent for large transit agencies. The maximum value for large transit agencies is 29.9 percent (Los Angeles). For medium-size transit systems, the mean value is 7.0 percent with a maximum of 16 percent (Tempe). For small transit systems, the mean value is 6.2 percent with a maximum of 33.3 percent (Concord, NC). Table 2-7 below shows the percentage of open work to total shifts for large, medium-size, small transit systems, and shuttles. 
Table 2-7

Basic System Information Statistics - Percentage of Open Work to Total Shifts

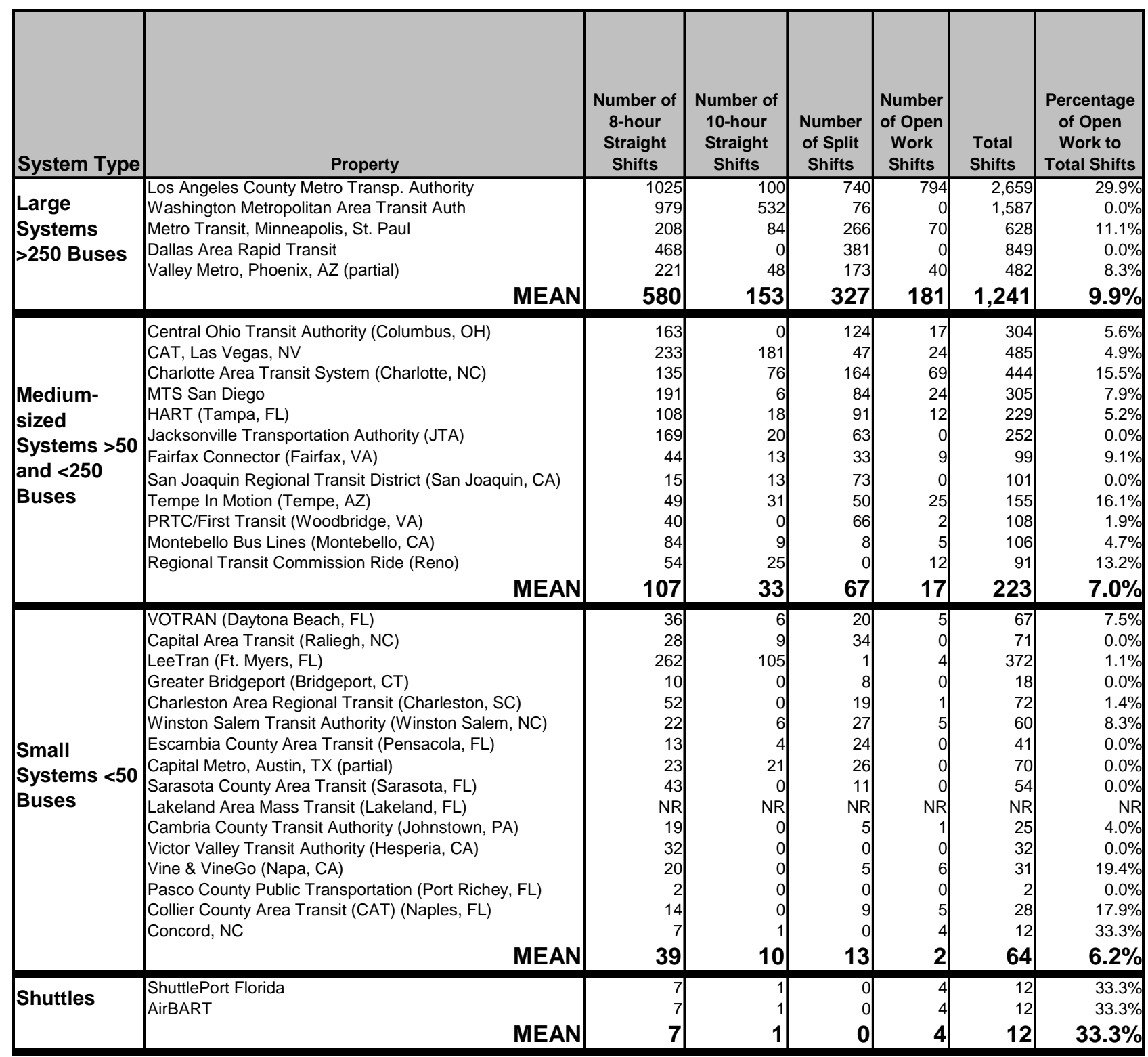




\section{Designated Extraboard Operators}

The following statistics apply to the designated extraboard for each of the system respondents to the survey.

Percentage of Full Time Extraboard Operators to Total Operators

This data set shows the first significant divergence between large, medium-size, and small transit agencies. In general, large transit agencies have a higher percentage of extraboard operators and smaller systems have a lower percentage in relation to total operators. For large transit agencies, the mean value is 19.6 percent with a maximum of 25.8 percent (Los Angeles). The mean value for medium-size systems is 12.1 percent with a maximum of 19.8 percent (Reno). For small transit systems, the mean value is 5.9 percent with a maximum of 18.5 percent (Daytona Beach). Shuttles have the lowest percentage of full time extraboard operators to total operators at a mean value of 2.3 percent and a maximum of 3.4 percent (AirBART).

\section{Percentage of Full Time Extraboard Operators to Full Time Operators}

This data set shows a similar divergence between system sizes. In large transit agencies, the mean value is 22.9 percent with a maximum of 34.4 percent (Los Angeles). For medium-size systems, the mean is 12.3 percent with a maximum of 20.5 percent (Reno), and for small transit agencies the mean value is 6.6 percent with a maximum of 18.7 percent (Daytona Beach). Shuttles have the lowest percentage of full time extraboard operators to total operators at a mean value of 2.8 percent and a maximum of 4.3 percent (AirBART).

\section{Percentage of Part Time Extraboard Operators to Total Operators}

This data set suggests that a vast majority of responding transit systems regardless of size do not employ part time extraboard operators. A total of 23 systems reported a value of 0 part time operators. Therefore, the mean values are 0.0 percent for large transit systems and shuttles, 1.9 percent for medium-size transit systems and 4.4 percent for small transit systems. 


\section{Percentage of Total Designated Extraboard $(F T+P T)$ to Total Operators}

Since the number of part time extraboard operators reported was so low for most systems, this data set is not significantly different than the percentage of Full Time extraboard Operators to Total Operators. The mean value for large systems is 19.6 percent with a maximum of 25.8 percent (Los Angeles). The mean value for medium size systems is 14.0 percent with a maximum of 23.4 percent (Reno) and the mean value for small systems is higher than the percentage of full time extraboard operators to total operators at 10.3 percent with a maximum of 28.6 percent (Naples). Shuttles have a mean value of 2.3 percent.

\section{Ratio of Total Designated Extraboard Operators to Open Work}

In general, most systems have more extraboard operators than open work, meaning that extraboard operators are used for other purposes in addition to filling open work. The mean value for large transit systems is 2.89 extraboard operators per piece of open work; the mean value for medium-size transit systems is 3.62 extraboard operators per piece of open work, and the mean value for small transit systems is 3.81 extraboard operators per piece of open work. The mean value for shuttles is 0.5 .

\section{Ratio of Total Extraboard Operators to Weekday Revenue Hours}

Of all the statistical data compiled in this section, the most consistent between different size systems is this particular ratio. For large transit agencies, the mean value is 0.04 , meaning that total extraboard operators are 4 percent of weekday revenue hours. For medium size and small systems, the ratio is 0.03 , meaning that total extraboard operators account for 3 percent of weekday revenue hours. This level of balance is useful to practitioners when examining the size of the board in relation to individual systems' weekday revenue hours.

Table 2-8 below displays statistical data for designated extraboard operators. 


\section{Table 2-8}

Basic System Information Statistics - Designated Extraboard Operators

\begin{tabular}{|c|c|c|c|c|c|c|c|c|c|c|}
\hline System Type & Property & $\begin{array}{c}\text { Designated } \\
\text { Full Time } \\
\text { Extraboard }\end{array}$ & \begin{tabular}{|c|} 
Designated \\
Part Time \\
Extraboard
\end{tabular} & $\begin{array}{c}\text { Total } \\
\text { Designated } \\
\text { Extra Board }\end{array}$ & $\begin{array}{c}\text { Percentage } \\
\text { of Full Time } \\
\text { Extra Board } \\
\text { Operators } \\
\text { to Total } \\
\text { Operators }\end{array}$ & $\begin{array}{c}\text { Percentage } \\
\text { of Full Time } \\
\text { Extra Board } \\
\text { Operators } \\
\text { to Total Full } \\
\text { Time } \\
\text { Operators }\end{array}$ & $\begin{array}{c}\text { Percentage } \\
\text { of Part Time } \\
\text { Extra Board } \\
\text { Operators to } \\
\text { Total } \\
\text { Operators }\end{array}$ & $\begin{array}{c}\text { Percentage of } \\
\text { Total } \\
\text { Designated } \\
\text { Extra Board } \\
\text { Operators to } \\
\text { Total } \\
\text { Operators }\end{array}$ & $\begin{array}{c}\text { Ratio of Total } \\
\text { Designated } \\
\text { Extra Board } \\
\text { Operators to } \\
\text { Open Work }\end{array}$ & $\begin{array}{c}\text { Ratio of Total } \\
\text { Extra Board } \\
\text { Operators to } \\
\text { Weekday } \\
\text { Revenue } \\
\text { Hours }\end{array}$ \\
\hline \multirow{6}{*}{$\begin{array}{l}\text { Large } \\
\text { Systems } \\
\text { >250 Buses }\end{array}$} & Los Angeles County Metro Transp. Authority & 1,000 & 0 & 1000 & $25.8 \%$ & $34.4 \%$ & $0.0 \%$ & $25.8 \%$ & 1.26 & 0.05 \\
\hline & Washington Metropolitan Area Transit Auth & 316 & 0 & 316 & $14.0 \%$ & $15.2 \%$ & $0.0 \%$ & $14.0 \%$ & N/A & 0.03 \\
\hline & Metro Transit, Minneapolis, St. Paul & 280 & 0 & 280 & $19.5 \%$ & $25.5 \%$ & $0.0 \%$ & $19.5 \%$ & 4.00 & 0.05 \\
\hline & Dallas Area Rapid Transit & 239 & 0 & 239 & $18.6 \%$ & $19.0 \%$ & $0.0 \%$ & $18.6 \%$ & $\mathrm{~N} / \mathrm{A}$ & 0.04 \\
\hline & Valley Metro, Phoenix, AZ (partial) & 136 & 0 & 136 & $19.9 \%$ & $20.5 \%$ & $0.0 \%$ & $19.9 \%$ & 3.40 & 0.05 \\
\hline & MEAN & 394 & 0 & 394.2 & $19.6 \%$ & $22.9 \%$ & $0.0 \%$ & $19.6 \%$ & 2.89 & 0.04 \\
\hline \multirow{13}{*}{$\begin{array}{l}\text { Medium- } \\
\text { sized } \\
\text { Systems >50 } \\
\text { and <250 } \\
\text { Buses }\end{array}$} & Central Ohio Transit Authority (Columbus, $\mathrm{OH}$ ) & 72 & 0 & 72 & $19.5 \%$ & $19.5 \%$ & $0.0 \%$ & $19.5 \%$ & 4.24 & 0.03 \\
\hline & CAT, Las Vegas, NV & 82 & 5 & 87 & $9.8 \%$ & $9.9 \%$ & $0.6 \%$ & $10.4 \%$ & 3.63 & 0.02 \\
\hline & Charlotte Area Transit System (Charlotte, NC) & 113 & 0 & 113 & $19.2 \%$ & $19.2 \%$ & $0.0 \%$ & $19.2 \%$ & 1.64 & 0.04 \\
\hline & MTS San Diego & 9 & 0 & 9 & $3.1 \%$ & $3.1 \%$ & $0.0 \%$ & $3.1 \%$ & 0.38 & 0.01 \\
\hline & HART (Tampa, FL) & 42 & 0 & 42 & $14.0 \%$ & $14.4 \%$ & $0.0 \%$ & $14.0 \%$ & 3.50 & 0.02 \\
\hline & Jacksonville Transportation Authority (JTA) & 46 & 0 & 46 & $12.4 \%$ & $12.7 \%$ & $0.0 \%$ & $12.4 \%$ & $\mathrm{~N} / \mathrm{A}$ & 0.02 \\
\hline & Fairfax Connector (Fairfax, VA) & 14 & 0 & 14 & $9.3 \%$ & $9.7 \%$ & $0.0 \%$ & $9.3 \%$ & 1.56 & 0.02 \\
\hline & San Joaquin Regional Transit District (San Joaquin, CA) & 31 & 0 & 31 & $18.2 \%$ & $18.5 \%$ & $0.0 \%$ & $18.2 \%$ & N/A & 0.03 \\
\hline & Tempe In Motion (Tempe, AZ) & 14 & 3 & 17 & $6.1 \%$ & $6.3 \%$ & $1.3 \%$ & $7.4 \%$ & 0.68 & 0.01 \\
\hline & PRTC/First Transit (Woodbridge, VA) & 18 & 6 & 24 & $13.8 \%$ & $14.4 \%$ & $4.6 \%$ & $18.5 \%$ & 12.00 & 0.04 \\
\hline & Montebello Bus Lines (Montebello, CA) & & 24 & 24 & $0.0 \%$ & $0.0 \%$ & $13.0 \%$ & $13.0 \%$ & 4.80 & 0.03 \\
\hline & Regional Transit Commission Ride (Reno) & 39 & 7 & 46 & $19.8 \%$ & $20.5 \%$ & $3.6 \%$ & $23.4 \%$ & 3.83 & 0.06 \\
\hline & MEAN & 40 & 4 & 43.75 & $12.1 \%$ & $12.3 \%$ & $1.9 \%$ & $14.0 \%$ & 3.62 & 0.03 \\
\hline \multirow{17}{*}{$\begin{array}{l}\text { Small } \\
\text { Systems }<50 \\
\text { Buses }\end{array}$} & VOTRAN (Daytona Beach, FL) & 20 & 1 & 21 & $18.5 \%$ & $18.7 \%$ & $0.9 \%$ & $19.4 \%$ & 4.20 & 0.04 \\
\hline & Capital Area Transit (Raliegh, NC) & & 0 & 4 & $3.5 \%$ & $3.7 \%$ & $0.0 \%$ & $3.5 \%$ & N/A & 0.01 \\
\hline & LeeTran (Ft. Myers, FL) & 8 & 0 & 8 & $6.1 \%$ & $6.7 \%$ & $0.0 \%$ & $6.1 \%$ & 2.00 & 0.01 \\
\hline & Greater Bridgeport (Bridgeport, CT) & & 0 & 0 & $0.0 \%$ & $0.0 \%$ & $0.0 \%$ & $0.0 \%$ & $\mathrm{~N} / \mathrm{A}$ & 0.00 \\
\hline & Charleston Area Regional Transit (Charleston, SC) & 7 & 0 & 7 & $7.1 \%$ & $7.1 \%$ & $0.0 \%$ & $7.1 \%$ & 7.00 & 0.01 \\
\hline & Winston Salem Transit Authority (Winston Salem, NC) & & 5 & 8 & $4.5 \%$ & $4.9 \%$ & $7.6 \%$ & $12.1 \%$ & 1.60 & 0.02 \\
\hline & Escambia County Area Transit (Pensacola, FL) & & 10 & 15 & $7.7 \%$ & $9.1 \%$ & $15.4 \%$ & $23.1 \%$ & $N / A$ & 0.05 \\
\hline & Capital Metro, Austin, TX (partial) & 5 & 0 & 5 & $6.7 \%$ & $7.1 \%$ & $0.0 \%$ & $6.7 \%$ & $\mathrm{~N} / \mathrm{A}$ & 0.01 \\
\hline & Sarasota County Area Transit (Sarasota, FL) & 14 & 0 & 14 & $12.4 \%$ & $12.4 \%$ & $0.0 \%$ & $12.4 \%$ & $\mathrm{~N} / \mathrm{A}$ & 0.04 \\
\hline & Lakeland Area Mass Transit (Lakeland, FL) & o & 0 & 0 & $0.0 \%$ & $0.0 \%$ & $0.0 \%$ & $0.0 \%$ & $N / A$ & 0.00 \\
\hline & Cambria County Transit Authority (Johnstown, PA) & 5 & 8 & 13 & $10.9 \%$ & $13.2 \%$ & $17.4 \%$ & $28.3 \%$ & 13.00 & 0.06 \\
\hline & Victor Valley Transit Authority (Hesperia, CA) & 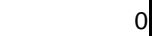 & 0 & 0 & $0.0 \%$ & $0.0 \%$ & $0.0 \%$ & $0.0 \%$ & $\mathrm{~N} / \mathrm{A}$ & 0.00 \\
\hline & Vine \& VineGo (Napa, CA) & 1 & 0 & 1 & $3.2 \%$ & $3.2 \%$ & $0.0 \%$ & $3.2 \%$ & 0.17 & 0.00 \\
\hline & Pasco County Public Transportation (Port Richey, FL) & & 0 & 2 & $3.9 \%$ & $3.9 \%$ & $0.0 \%$ & $3.9 \%$ & $\mathrm{~N} / \mathrm{A}$ & 0.13 \\
\hline & Collier County Area Transit (CAT) (Naples, FL) & 0 & 10 & 10 & $0.0 \%$ & $0.0 \%$ & $28.6 \%$ & $28.6 \%$ & 2.00 & 0.06 \\
\hline & Concord, NC & & 0 & & $10.0 \%$ & $15.4 \%$ & $0.0 \%$ & $10.0 \%$ & 0.50 & 0.02 \\
\hline & MEAN & 5 & 2 & 6.88 & $5.9 \%$ & $6.6 \%$ & $4.4 \%$ & $10.3 \%$ & 3.81 & 0.03 \\
\hline \multirow{3}{*}{ Shuttles } & ShuttlePort Florida & 2 & 0 & 2 & $1.2 \%$ & $1.3 \%$ & $0.0 \%$ & $1.2 \%$ & 0.50 & 0.00 \\
\hline & AirBART & & 0 & & $3.4 \%$ & $4.3 \%$ & $0.0 \%$ & $3.4 \%$ & 0.50 & 0.00 \\
\hline & MEAN & 2 & 0 & 2.00 & $2.3 \%$ & $2.8 \%$ & $0.0 \%$ & $2.3 \%$ & 0.50 & 0.00 \\
\hline
\end{tabular}




\section{Labor Agreement}

The survey asked systems to provide information about labor agreements in the form of yes/no questions. Agencies were asked if they have a collective bargaining agreement, if the agreement addresses limitations in the number or percentage of full time and part time employees, and if the agreement addresses policies, rules, deployments, seniority and other provisions related to the extraboard.

Of the large transit agencies, two responded affirmatively to all three questions: WMATA serving Washington, DC, and Metro Transit in Minneapolis/St. Paul. Of the medium systems, eight systems responded affirmatively to all three questions: Charlotte, HART in Tampa, the Jacksonville Transportation Authority, Fairfax Connector, San Joaquin, Tempe, First Transit Woodbridge, and Reno. Of the small systems, there were five systems responding affirmatively to all three questions: Raleigh, Charleston, WinstonSalem, ECAT (Pensacola), and Cambria (Johnstown, PA).

For the large transit agencies, all of them have a collective bargaining agreement and all have agreements that address policies and rules related to the extraboard. For the medium size transit agencies, all 12 have a collective bargaining agreement and 10 (92 percent) have agreements addressing policies and rules related to the extraboard.

For the small systems, 11 (69 percent) have a collective bargaining agreement and 10 systems (63 percent) have agreements that address policies and rules related to the extraboard. Shuttles did not respond affirmatively to any of the labor agreement questions. Table 2-9 below shows the number of systems responding affirmatively to questions regarding labor agreements.

Table 2-9

Labor Agreements

\begin{tabular}{|l|r|r|r|r|r|r|r|r|}
\hline \multicolumn{1}{|c|}{ Agencies Responding Affirmatively: } & $\begin{array}{c}\text { Large } \\
\text { Systems } \\
\text { (N=5) }\end{array}$ & $\begin{array}{c}\text { Percent of } \\
\text { Total Survey } \\
\text { Respondents }\end{array}$ & $\begin{array}{c}\text { Medium- } \\
\text { Size } \\
\text { Systems } \\
\text { (N=12) }\end{array}$ & $\begin{array}{c}\text { Percent of } \\
\text { Total Survey } \\
\text { Respondents }\end{array}$ & $\begin{array}{c}\text { Small } \\
\text { Systems } \\
\text { (N=16) }\end{array}$ & $\begin{array}{c}\text { Percent of } \\
\text { Total Survey } \\
\text { Respondents }\end{array}$ & $\begin{array}{c}\text { Percent of } \\
\text { Total Survey } \\
\text { Respondtles } \\
\text { S=2) }\end{array}$ \\
\hline Collective Bargaining Agreement & 5 & $100.0 \%$ & 12 & $100.0 \%$ & 11 & $68.8 \%$ & 0 & $0.0 \%$ \\
\hline $\begin{array}{l}\text { Agreement addresses limitations ratio of full- } \\
\text { time to part-time employees }\end{array}$ & 2 & $40.0 \%$ & 8 & $66.7 \%$ & 5 & $31.3 \%$ & 0 & $0.0 \%$ \\
\hline $\begin{array}{l}\text { Agreement addresses policies, rules, } \\
\text { deployments, seniority, overtime and/or other } \\
\text { provisions related to extra board } \\
\text { management }\end{array}$ & & & & & & & & \\
\hline
\end{tabular}




\section{Absenteeism and Attrition}

Absenteeism and attrition are two factors that can greatly influence the size of the extraboard and its deployment. Absenteeism is divided into two categories: scheduled leave and unscheduled leave. Obviously, unscheduled leave is the most variable factor for any operations department to address. Attrition refers to employees terminating employment through resignation, termination, or retirement. Therefore, the survey was designed to determine the degree to which systems attempt to plan for these factors by determining rates of absenteeism and attrition.

Of the large systems, only one system has an established rate for all three (scheduled leave, unscheduled leave, and attrition): Metro Transit Minneapolis/St. Paul. Of the medium size systems, three transit systems have an established rate for all three: COTA (Columbus), MTS San Diego, and San Joaquin. Of the small transit systems, four systems have an established rate for all three: Daytona Beach, Raleigh, Winston Salem, and Sarasota. This data set is helpful in selecting a system for case study in developing a guide for transit agencies. Table 2-10 below displays the total systems that have an established rate for at least one of the three categories.

Table 2-10

Absenteeism and Attrition

\begin{tabular}{|c|c|c|c|c|c|c|c|c|}
\hline Agencies Responding Affirmatively: & $\begin{array}{l}\text { Large } \\
\text { Systems } \\
(\mathrm{N}=5)\end{array}$ & $\begin{array}{l}\text { Percent of } \\
\text { Total Survey } \\
\text { Respondents }\end{array}$ & $\begin{array}{c}\text { Medium- } \\
\text { Size } \\
\text { Systems } \\
(\mathrm{N}=12) \\
\end{array}$ & $\begin{array}{l}\text { Percent of } \\
\text { Total Survey } \\
\text { Respondents }\end{array}$ & \begin{tabular}{|c|} 
Small \\
Systems \\
$(\mathrm{N}=16)$ \\
\end{tabular} & \begin{tabular}{|c|} 
Percent of \\
Total Survey \\
Respondents
\end{tabular} & Shuttles & $\begin{array}{c}\text { Percent of } \\
\text { Total Survey } \\
\text { Respondents } \\
(\mathrm{N}=2) \\
\end{array}$ \\
\hline $\begin{array}{l}\text { Established rate of attrition including } \\
\text { resignation, termination and/or } \\
\text { retirement }\end{array}$ & 3 & $60.0 \%$ & 5 & $41.7 \%$ & 7 & $43.8 \%$ & 0 & $0.0 \%$ \\
\hline $\begin{array}{l}\text { Established rate of scheduled leave } \\
\text { including vacation, training, and/or } \\
\text { union business }\end{array}$ & 2 & $40.0 \%$ & 11 & $91.7 \%$ & 11 & $68.8 \%$ & 1 & $50.0 \%$ \\
\hline $\begin{array}{l}\text { Established rate of unscheduled leave } \\
\text { including sick, jury duty and/or } \\
\text { administrative leave }\end{array}$ & 3 & $60.0 \%$ & 4 & $33.3 \%$ & 7 & $43.8 \%$ & 1 & $50.0 \%$ \\
\hline
\end{tabular}




\section{Scheduling}

The purpose of this section of the survey was to determine whether systems use automated scheduling, whether automated scheduling software assists in determining the extraboard requirement and, if so, does the automated scheduling software accurately calculate extraboard requirements in terms of the agency's actual number of extraboard operators. Interestingly, no system of any size with automated scheduling capability has automated scheduling software capable of determining the extraboard requirement. Consultations with Trapeze and Hastus, two large distributors of automated scheduling software, indicated that their products are not configured to address this particular labor requirement. Therefore, this survey shows that automated scheduling software is not deemed a factor in extraboard management. Table 2-11 below shows the responses to this section of the survey.

Table 2-11

\section{Scheduling}

\begin{tabular}{|c|c|c|c|c|c|c|c|c|}
\hline Agencies Responding Affirmatively: & $\begin{array}{l}\text { Large } \\
\text { Systems } \\
(\mathrm{N}=5)\end{array}$ & $\begin{array}{l}\text { Percent of } \\
\text { Total Survey } \\
\text { Respondents } \\
\end{array}$ & $\begin{array}{l}\text { Medium- } \\
\text { Size } \\
\text { Systems } \\
(\mathrm{N}=12) \\
\end{array}$ & $\begin{array}{l}\text { Percent of } \\
\text { Total Survey } \\
\text { Respondents }\end{array}$ & \begin{tabular}{|c|} 
Small \\
Systems \\
$(\mathrm{N}=16)$ \\
\end{tabular} & \begin{tabular}{|c|} 
Percent of \\
Total Survey \\
Respondents
\end{tabular} & Shuttles & $\begin{array}{c}\text { Percent of } \\
\text { Total Survey } \\
\text { Respondents } \\
(\mathrm{N}=2) \\
\end{array}$ \\
\hline $\begin{array}{l}\text { Agency uses automated scheduling } \\
\text { for master schedules, runcuts and/or } \\
\text { interlines }\end{array}$ & 5 & $100.0 \%$ & 10 & $83.3 \%$ & 5 & $31.3 \%$ & 0 & $0.0 \%$ \\
\hline $\begin{array}{l}\text { Scheduling software calculates } \\
\text { number of extra board operators } \\
\text { required }\end{array}$ & 0 & $0.0 \%$ & 0 & $0.0 \%$ & 0 & $0.0 \%$ & 0 & $0.0 \%$ \\
\hline $\begin{array}{l}\text { Scheduling software accurately } \\
\text { calculates number of extra board } \\
\text { operators required }\end{array}$ & 0 & $0.0 \%$ & 0 & $0.0 \%$ & 0 & $0.0 \%$ & 0 & $0.0 \%$ \\
\hline $\begin{array}{l}\text { Established number of extra board } \\
\text { operators needed for each bid }\end{array}$ & 5 & $100.0 \%$ & 8 & $66.7 \%$ & 6 & $37.5 \%$ & 2 & $100.0 \%$ \\
\hline
\end{tabular}




\section{Operations}

Previous sections of the survey attempted to address factors that impact extraboard requirements in terms of the need, size, and the availability of this labor force subset. However, regardless of the size of the extraboard, there are operational factors that can be impacted by work rules and deployment of extraboard operators. In other words, all issues surrounding the extraboard must be operationalized in such a manner that the dispatch function of operations can insure that the agency makes pull-out and reliefs each service day. The purpose of this section of the survey was to explore the operational factors that practitioners (specifically dispatchers) face in the deployment of the extraboard.

For non-practitioners, it is helpful to define the important terms used in this section of the survey.

The Order- During the bid/mark-up period, operators bid the extraboard in seniority order to form a list of available extraboard operators. The order of seniority of extraboard operators does not change. Once the list has been established at the end of the bid, the order refers to one of two systems by which dispatchers can assign runs to extraboard operators over a period of time. The two systems are fixed and rotating. When the order of the extraboard is fixed, it means that operators bid the extraboard based on seniority and work is offered from dispatch in the same seniority order over time. When the order is rotating, it means that work offered by dispatch to extraboard operators changes on a periodic basis (daily, weekly, or by bid period) regardless of the seniority of the operator. A rotating order is considered to be more equitable because it means that desirable and undesirable pieces of work will be periodically distributed to all extraboard operators.

Hold Down Assignments - refers to those runs that are temporarily open due to the fact that the regular operator who bid the assignment is not available to perform because of scheduled or unscheduled absence. Hold down assignments are those that must be preserved for the regular operator in the event that he/she is unable to perform it for a period of time but is expected to return to work whether within days, weeks, or months.

Known Open Work- refers to those operator runs that become unfilled because of a scheduled leave such as vacation, union business, training, or non-recurring special assignment. The dispatcher would have previous knowledge that this work must be covered. 
Unanticipated Open Work - refers to those operator runs that become unfilled because of an unscheduled event such as a miss-out, illness, or other circumstance that was not previously known to the dispatcher.

Special Work Assignments - refers to any assignments that are non-recurring and are not part of the normal bid/mark-up process and therefore have no regular bus operator performing the run.

Option to Pass - refers to the degree to which extraboard operators have discretion in accepting or declining runs offered by dispatch.

Miss-out - refers to an operator who either arrives too late or no-shows the run altogether such that the run becomes open and/or unfilled. This is the same type of unanticipated event wherein the dispatcher would not have previous knowledge of the vacancy of the run.

\section{Order}

For large and medium-sized systems, the order of the extraboard is generally rotating on a daily basis. For small systems, the order of the extraboard is split between half the responding systems having a fixed and half of the responding systems having a rotating order. For all size systems with a rotating order, the prevalence for rotating the order is on a daily basis.

\section{Operators Allowed to Pick}

For large systems, three of the five systems allow operators to pick known open work and unanticipated open work. Two systems allow operators to pick hold downs and special work assignments.

For medium-size systems, 67 percent allow operators to pick hold downs; however, only 50 percent of responding transit agencies allow operators to pick known open work and unanticipated open work. Five systems (42 percent) allow operators to pick special work assignments. 


\section{Operators Have Option to Pass}

For the large systems, DART Dallas allows operators to pass on hold downs and special work assignments. Valley Metro Phoenix allows operators to pass on unanticipated open work.

For the medium-size systems, two systems allow operators to pass for known open work and unanticipated open work. Three systems allow operators to pass on special work assignments. Only one system allows operators to pass for hold downs.

For small systems, five systems allow operators to pass on hold downs and there are four systems that allow operators to pass for known open work, unanticipated open work, and special assignments.

\section{Operators Do Not Have Option to Pass}

For large systems, all five do not allow operators the option to pass on unanticipated open work and four of the five systems do not allow operators the option to pass on known open work. This same prevalence exists for medium sized systems.

Eleven of the 12 medium-size systems (92 percent) do not allow operators the option to pass on unanticipated open work. Ten of 12 systems (83 percent) do not allow operators the option to pass on known open work and special work assignments. Five systems (42 percent) do not allow operators the option to pass on hold downs.

Though not as prevalent as the medium-size and large systems, small systems show a preference for not allowing operators the option to pass on assignments. Ten of 16 systems (63 percent) do not allow operators to pass on known open work and unanticipated open work. Nine systems (56 percent) do not allow operators the option to pass on special assignments and 7 systems (44 percent) do not allow operators the option to pass on hold downs.

\section{Miss Outs}

Two of the five large systems allow regular operators to work the extraboard when they have a miss-out (arrive too late to perform regular shift). Nine of the 12 medium size 
systems (75 percent) allow this practice. Eleven of 16 small systems (69 percent) also allow this practice.

Table 2-12 below displays the survey respondents and their operations practices for assigning extraboard operators. 
Table 2-12

Operations Practice - Assignment of Extraboard Operators

\begin{tabular}{|c|c|c|c|c|c|c|c|c|}
\hline Agencies Responding Affirmatively: & $\begin{array}{c}\text { Large } \\
\text { Systems } \\
(\mathrm{N}=5)\end{array}$ & $\begin{array}{l}\text { Percent of } \\
\text { Total Survey } \\
\text { Respondents }\end{array}$ & $\begin{array}{l}\text { Medium- } \\
\text { Size } \\
\text { Systems } \\
(\mathrm{N}=12)\end{array}$ & $\begin{array}{l}\text { Percent of } \\
\text { Total Survey } \\
\text { Respondents }\end{array}$ & $\begin{array}{c}\text { Small } \\
\text { Systems } \\
(\mathrm{N}=16)\end{array}$ & $\begin{array}{c}\text { Percent of } \\
\text { Total Survey } \\
\text { Respondents }\end{array}$ & Shuttles & $\begin{array}{c}\text { Percent of } \\
\text { Total Survey } \\
\text { Respondents } \\
(\mathrm{N}=2)\end{array}$ \\
\hline $\begin{array}{l}\text { Order of Extra Board Assignment is Fixed, or the } \\
\text { same over time }\end{array}$ & 1 & $20.0 \%$ & 2 & $16.7 \%$ & 8 & $50.0 \%$ & 2 & $100.0 \%$ \\
\hline $\begin{array}{l}\text { Order of Extra Board Assignment is a Rotating } \\
\text { System }\end{array}$ & 5 & $100.0 \%$ & 9 & $75.0 \%$ & 8 & $50.0 \%$ & 0 & $0.0 \%$ \\
\hline \multicolumn{9}{|l|}{$\begin{array}{l}\text { If the order of Extra Board assignment is rotating, } \\
\text { the frequency of change is: }\end{array}$} \\
\hline Daily] & 4 & $80.0 \%$ & 10 & $83.3 \%]$ & 6 & $37.5 \%$ & 0 & $0.0 \%$ \\
\hline Weekly & 3 & $60.0 \%$ & 1 & $8.3 \%$ & 2 & $12.5 \%$ & 0 & $0.0 \%$ \\
\hline By pick or mark-up period & 0 & $0.0 \%$ & 2 & $16.7 \%$ & 1 & $6.3 \%$ & 0 & $0.0 \%$ \\
\hline \multicolumn{9}{|l|}{$\begin{array}{l}\text { Work assignments that Extra Board operators are } \\
\text { allowed to pick include the following: }\end{array}$} \\
\hline Assignments held down for more than one day & 2 & $40.0 \%$ & 8 & $66.7 \%$ & 7 & $43.8 \%$ & 2 & $100.0 \%$ \\
\hline Known open work posted for the next day & 3 & $60.0 \%$ & 6 & $50.0 \%$ & 7 & $43.8 \%$ & 2 & $100.0 \%$ \\
\hline Unanticipated open work (e.g. miss-outs) & 3 & $60.0 \%$ & 6 & $50.0 \%$ & 7 & $43.8 \%$ & 1 & $50.0 \%$ \\
\hline Other (e.g. special work assignments) & 2 & $40.0 \%$ & 5 & $41.7 \%$ & 4 & $25.0 \%$ & 2 & $100.0 \%$ \\
\hline Not Applicable] & 2 & $40.0 \%$ & 1 & $8.3 \%$ & 4 & $25.0 \%$ & 0 & $0.0 \%$ \\
\hline \multicolumn{9}{|l|}{$\begin{array}{l}\text { Work Assignments assigned by Dispatch for } \\
\text { which operators have option to pass }\end{array}$} \\
\hline Assignments held down for more than one day & 1 & $20.0 \%$ & 1 & $8.3 \%$ & 5 & $31.3 \%$ & 1 & $50.0 \%$ \\
\hline Known open work posted for the next day & 0 & $0.0 \%$ & 2 & $16.7 \%$ & 4 & $25.0 \%$ & 1 & $50.0 \%$ \\
\hline Unanticipated open work (e.g. miss-outs) & 1 & $20.0 \%$ & 2 & $16.7 \%$ & 4 & $25.0 \%$ & 0 & $0.0 \%$ \\
\hline Other (e.g. special work assignments) & 1 & $20.0 \%$ & 3 & $25.0 \%$ & 4 & $25.0 \%$ & 0 & $0.0 \%$ \\
\hline Not Applicable & 3 & $60.0 \%$ & 5 & $41.7 \%$ & 7 & $43.8 \%$ & 1 & $50.0 \%$ \\
\hline \multicolumn{9}{|l|}{$\begin{array}{l}\text { Work Assignments assigned by Dispatch for } \\
\text { which operators do not have option to pass }\end{array}$} \\
\hline Assignments held down for more than one day & 2 & $40.0 \%$ & 5 & $41.7 \%$ & 7 & $43.8 \%$ & 2 & $100.0 \%$ \\
\hline Known open work posted for the next day & 4 & $80.0 \%$ & 10 & $83.3 \%$ & 10 & $62.5 \%$ & 2 & $100.0 \%$ \\
\hline Unanticipated open work (e.g. miss-outs) & 5 & $100.0 \%$ & 11 & $91.7 \%$ & 10 & $62.5 \%$ & 2 & $100.0 \%$ \\
\hline Other (e.g. special work assignments) & 3 & $60.0 \%$ & 10 & $83.3 \%$ & 9 & $56.3 \%$ & 2 & $100.0 \%$ \\
\hline Not Applicable & 0 & $0.0 \%$ & 1 & $8.3 \%$ & 1 & $6.3 \%$ & 0 & $0.0 \%$ \\
\hline Miss-outs are eligible to work Extra Board & 2 & $40.0 \%$ & 9 & $75.0 \%$ & 11 & $68.8 \%$ & 1 & $50.0 \%$ \\
\hline
\end{tabular}




\section{Operations - Other}

In the final part of the Operations section of the survey, respondents were asked to provide information on the process used to determine extraboard size whether the process for individual systems is based on factors included in the survey or other factors unique to the transit agency. Next, systems were asked to provide other factors, tools, or practices used in extraboard management that may not be covered in the survey instrument. In responding to these questions, some systems provided a narrative for how they determine extraboard requirements. Some systems provided a list of the factors in the survey that they use. Other systems provided a degree of detail on their process in the form of spreadsheets. One of these detailed spreadsheets will be discussed later in this section.

Below is a list of unedited responses to the first question regarding the process used for extraboard determination and is presented regardless of transit agency size.

Washington Metropolitan Area Transit Authority (WMATA)

To cover controllable and uncontrollable absenteeism.

Metro Transit, Minneapolis, St. Paul

A model that uses historical data and inputs

Valley Metro, Phoenix, AZ (partial)

Scheduled vacations, estimated number off by medical leave, prescheduled personal holidays, estimated authorized time, and training needs.

Central Ohio Transit Authority (Columbus, $\mathrm{OH}$ )

The total number of regular runs (daily + Sat and Sun) in a week is divided by 5 giving the number of operators needed. This number is further adjusted by recent absentee rate percentage.

CAT, Las Vegas, NV

History, the system in use is code based. In other words each transaction recorded is coded and we track and trend each code, which are divided into groups such as Operational, non-operational, etc. 
Charlotte Area Transit System (Charlotte, NC)

Percentage of runs/absentee rate

MTS San Diego

As stated previously, we have not had an extraboard in the past two years due to severe driver shortage in San Diego. If we were at full compliment we would have 9 extraboard positions for the 281 pieces of work.

San Joaquin Regional Transit District (San Joaquin, CA)

History plus the amount of unhooked work (extraboard pieces)

Montebello Bus Lines (Montebello, CA)

Comparing number of operators with open pieces of work and trend of sick/medical leave, scheduled/unscheduled leave to determine the number of operators for each bid period.

Regional Transit Commission Ride (Reno)

The number of extraboard operators is determined by many factors including work not picked by bid operators, average number of operators off each day and training coverage needs.

VOTRAN (Daytona Beach, FL)

Amount of unscheduled work, vacations, illnesses, other absentees

Capital Area Transit (Raleigh, NC)

Based on miss-outs, extra work and to keep down OT.

LeeTran (Ft. Myers, FL)

Average number of operators absent.

Winston Salem Transit Authority (Winston Salem, NC)

10 to $15 \%$ of operators, absentees determine the number of extraboard operator.

Capital Metro, Austin, TX (partial)

Goal is $10 \%$ but allow $15 \%$. 
Sarasota County Area Transit (Sarasota, FL)

SCAT attempts to provide a 15\% extraboard.

Cambria County Transit Authority (Johnstown, PA)

We permit 7 positions for scheduled days off out of the 13 available extraboard (FT \& PT). We average 3 Reporters per weekday and account for extraboard days off.

Pasco County Public Transportation (Port Richey, FL)

Two scheduled positions and additional openings covered by Paratransit drivers.

Collier County Area Transit (CAT) (Naples, FL)

An annual review is conducted based on attendance, holiday, vacation, etc. a calculation is made to determine the part time extraboard drivers.

Concord, NC

One A.M. and one P.M. extraboard position that is bid along with the rest of the runs. This positions fills in where needed.

\section{ShuttlePort Florida}

Average daily call outs.

\section{AirBART}

Coverage for pre-approved and forecast absences per day.

Only four respondents answered the second part of the question regarding other factors, tools or practices used in extraboard management that are not covered in the survey. Those four responses are provided below.

Central Ohio Transit Authority (Columbus, $\mathrm{OH}$ )

We have provisions for operators to give away their overtime assignments, sign up for voluntary assignments, and waive their 8 hour spread.

\section{CAT, Las Vegas, NV}

Trend analysis, seasonal changes and tourism based, large conventions can call for more extraboard to be assigned along with significant special events such as NASCAR Race, which pulls $100+$ buses on race day. 


\section{MTS San Diego}

Although we do not have an extraboard at this time, we do have client mandated standby buses which are bid in the general run shake up. To complete that weekly work schedule the drivers perform standby services which operate somewhat as an extraboard. They are included in the 290 positions above.

\section{Montebello Bus Lines (Montebello, CA)}

Put up an extra-work sign up sheet for operators who would like to work overtime when there is shortage of bodies to fulfill all open runs.

\section{DART - Dallas Area Rapid Transit, Dallas, TX}

Dallas provided an ongoing worksheet used to determine extraboard staffing that NCTR researchers found especially intriguing. The worksheet determines extraboard needs using actual tallies of absenteeism and attrition by month and then, based on current staffing, calculates the rate of recruiting that is necessary to maintain optimum operator staffing levels. Categories of absenteeism include:

- Unscheduled absences

- FMLA

- Alternative duty

- Scheduled absences

- Non-operating function

- Operator training and

- Union business

The worksheet calculates the number of absences for each one of these categories by month and then establishes a rolling trend and average over time. Categories of attrition (turnover) include:

- Terminations

- Promotions

- Transfers to train

Optimal bus forecast includes the optimal number of full-time and part-time operators. Current staffing then provides a value in relation to the optimal staffing level. Finally, the recruitment section provides the total number of students in training scheduled for 
graduation, and the required recruiting levels for full-time and part-time operators. Based on examining the worksheet in electronic format, further clarification will be necessary from DART to ascertain the manner in which some data sets are calculated. However, this worksheet is especially valuable because it could be universally applied to all transit systems. Table 2-13 below displays the DART worksheet. 
Table 2-13

DART Worksheet - Extraboard Staffing

MARCH 2007 OPTIMAL OPERATOR REPORT - THREE MONTH ROLLING AVERAGE

\begin{tabular}{|c|c|c|c|c|c|c|c|c|c|c|c|c|c|c|c|}
\hline BUS & & & & & & & & & & & & & Project & & \\
\hline & Mar & Apr & May & June & July & Aug & Sept & Oct & Nov & Dec & Jan & Feb & Apr & & \\
\hline TOTAL FTE BLOCKS OF WORK - FULL TIME & 1,010 & 1,010 & 1,010 & 1,010 & 1,010 & 1,010 & 1,008 & 1,008 & 1,008 & 1,008 & 1,008 & 1,008 & 1,016 & & \\
\hline TOTAL FTE BLOCKS OF WORK - PART TIME & 30 & 30 & 30 & 30 & 30 & 30 & 26 & 26 & 26 & 26 & 26 & 26) & 26 & & from planning department \\
\hline Extraboard Requirement" & & & & & & & & & & & & & Trend & Avg & \\
\hline Unscheduled Absences & 86 & 91 & 88 & 76 & 93 & 90 & 82 & 87 & 87 & 121 & 98 & 102 & 911 & 92 & \\
\hline FMLA (all categories - using VAC/Sick/LWP) & 12 & 11 & 9 & 11 & 12 & 11 & 9 & 10 & 11 & 12 & 11 & 11 & 111 & 11 & \\
\hline Alternative Duty & 22 & 20 & 20 & 15 & 13 & 15 & 20 & 23 & 23 & 3 & 16 & 14 & 18 & 17 & \\
\hline Scheduled Absences & 108 & 109 & 106 & 160 & 104 & 97 & 90 & 85 & 129 & 97 & 104 & 110 & 110 & 108 & \\
\hline Non-operating Function & 3 & 5 & 3 & 8 & 3 & 6 & 4 & 4 & 11 & 4 & 6 & 7 & 6 & 5 & \\
\hline Operator Training & 7 & 7 & 0 & 12 & 15 & 23 & 9 & 9 & 13 & 7 & 10 & 10 & 10 & 10 & \\
\hline Union Business & 3 & 3 & 3 & 4 & 2 & 3 & 3 & 2 & 3 & 2 & 3 & 3 & 3 & 3 & \\
\hline TOTAL EXTRABOARD REQUIREMENT & 241 & 247 & 230 & 285 & 242 & 245 & 216 & 220 & 278 & 246 & 248 & 257 & 249 & 246 & \\
\hline PERCENTAGE OF EXTRABOARD & $19.5 \%$ & $20.2 \%$ & $19.4 \%$ & $22.9 \%$ & $19.6 \%$ & $20.0 \%$ & $18.1 \%$ & $18.4 \%$ & $22.3 \%$ & $20.4 \%$ & $20.4 \%$ & $20.9 \%$ & $20.3 \%$ & $20.1 \%$ & $\begin{array}{l}\text { Calculation of \% for the extraboard is the } \\
\text { extraboard requirements + total turnover / FT } \\
\text { optimal bus forecast. }\end{array}$ \\
\hline Turnover - \# as of end of mth" & & & & & & & & & & & & & & & \\
\hline Terminations & 3 & 9 & 14. & 14 & 4 & 7 & 6 & 8 & 11 & 11 & 10 & 9 & 9 & 9 & \\
\hline Promotions & 0 & 0 & 0 & 0 & 0 & 0 & of & 0 & 0 & 2 & 1 & 1 & of & - & \\
\hline Transfers to train & of & of & 0 & 0 & of & of & of & 0 & of & 0 & of & o & 0 & o & \\
\hline Total Turnover & 3 & 9 & 14 & 14 & 4 & 7 & 6 & 8 & 11 & 13 & 11 & 10 & g & 9 & \\
\hline Transfers from Train ${ }^{*}$ & & & & & of & of & 의 & 의 $12-1-1$ & of & 0 & of & of & 0 & 0 & \\
\hline PERCENTAGE OF TURNOVER & $0.2 \%$ & $0.7 \%$ & $1.2 \%$ & $1.2 \%$ & $0.3 \%$ & $0.6 \%$ & $0.5 \%$ & $0.7 \%$ & $0.9 \%$ & $1.1 \%$ & $0.9 \%$ & $0.8 \%$ & $0.7 \%$ & $0.7 \%$ & $\begin{array}{l}\text { The turnover \% is determined by dividing the } \\
\text { total turnover/current staffing }\end{array}$ \\
\hline Optimal Bus Forecast" & & & & & & & & & & & & & & & \\
\hline Full-Time Bus Operators & 1,254 & 1,266 & 1,254 & 1,309 & 1,256 & 1,262 & 1,230 & 1,236 & 1,297 & 1,267 & 1,267 & 1,275 & 1,274 & 1,271 & \\
\hline Part-Time Bus Operators & 30] & 30 & 30 & 30 & 30 & 30 & 26 & 26 & 26 & 26 & 26 & 26] & $26]$ & 26 & \\
\hline TOTAL OPTIMAL BUS FORECAST & 1,284 & 1,296 & 1,284 & 1,339 & 1,286 & 1,292 & 1,256 & 1,262 & 1,323 & 1,293 & 1,293 & 1,301 & 1,300 & 1,297 & $\begin{array}{l}\text { totaling the blocks of work }+ \text { total extraboard } \\
+ \text { total turnover }\end{array}$ \\
\hline Current Staffing & & & & & & & & & & & & & & & \\
\hline Full-Time & 1,206 & 1,217 & 1,201 & 1,199 & 1,203 & 1,201 & 1,206 & 1,206 & 1,223 & 1,195 & 1,209 & 1,209 & 1,217 & 1,217 & previous month) \\
\hline Part-Time & 25 & 25 & 25 & 27 & 27. & 25 & 26 & 25 & 25 & 26 & 26 & 26. & 26. & 26 & 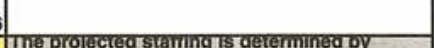 \\
\hline TOTAL CURRENT STAFFING & 1,231 & 1,242 & 1,226 & 1,226 & 1,230 & 1,226 & 1,232 & 1,231 & 1,248 & 1,221 & 1,235 & 1,235 & 1,243 & 1,243 & $\begin{array}{l}\text { adding the previous month }+ \text { the students } \\
\text { still in training at the end of last month }\end{array}$ \\
\hline Students in training, graduating next Month & 9 & व & 15 & 12 & 12 & 7 & 9 & 12 & 36 & 18 & 9 & 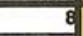 & & & \\
\hline REQUIRED RECRUITMENT LEVEL - FULL TIME & 48. & 49 & 53 & 110 & 53 & 61. & 24. & 30 & 74 & 72 & 58 & 66 & 57 & 54 & \\
\hline REQUIRED RECRUITMENT LEVEL - PART TIME & | & & & & & 5] & & & & 0 & 0 & 0 & of & & $\begin{array}{l}\text { by subtracting the current staffing from the } \\
\text { optimal bus forecast }\end{array}$ \\
\hline $\begin{array}{l}\text { Projected hires in current month (hired or cleared to hire } \\
\text { as if report date) }\end{array}$ & & & & & & & & & & & & & 9 & & \\
\hline
\end{tabular}




\section{SECTION THREE \\ Case Study and Guide For Extraboard Management}

\section{INTRODUCTION}

Based on the literature review, survey, and the stated goal of this study to provide practitioners with a useful tool in determining extraboard requirements, CUTR selected DART as the case study to achieve project goals. As discussed in the previous section, DART has an instrument that projects the optimal bus operator forecast for the agency that includes a component determining the extraboard. NCTR researchers conducted a site visit to DART and the staff assisted in the development of this section that includes a Detailed Analysis of the DART Optimal Operator Report, an Application of the DART Optimal Operator Report to a Florida Transit Agency, and finally Implications for All Transit Agencies.

\section{DART SITE VISIT}

CUTR had supplied DART with a copy of the first technical memorandum for this study prior to the site visit and asked staff to provide some insights into the results of the survey. DART staff indicated that extraboard requirements for large systems will always be driven by work rules and the labor contract. Therefore, mean values would be different between large and some medium transit agencies while small transit agencies are unlikely to be bound by labor contracts. Also, larger transit agencies would be more likely to offer larger benefits packages than smaller transit agencies.

The discussion then turned to the DART Optimal Operator Report. Staff indicated that extraboard sizing was a by-product of the overall organizational objective to determine optimal staffing for bus operations. In previous years, there had been a desire in the financial sector of the agency to determine bus operator staffing based on FTE units that would fulfill the needed blocks of operator work. In addition, Human Resources did not have a firm grasp on how the Operations Department determined its operator needs when requesting recruitment of bus operators at any given time. As a whole, the organization needed to address issues that were faced by all these departments (Finance, Operations, Service Planning, and Human Resources) in managing the transit agency. 
As a result, a task force was formed to develop an instrument that all departments and senior management of the agency could understand and use for management purposes. This task force worked for many months to determine the data collection needs and variables that had to be accounted for in determining bus operator staffing levels. In subsequent years, the instrument itself was refined based on trends in data and other organizational circumstances. The Information Technology Department of DART was able to configure SQL/Crystal reports that draw from the payroll system in order to provide inputs for the Optimal Operator Report.

DART staff indicated that the organization had spent considerable time, effort, and resources to develop and refine the instrument over a period of years and that all organizational objectives had been met. Staff indicated that they are proud of the instrument because it is formatted such that multiple audiences can read and understand it. This was the same conclusion that CUTR reached and this is why this study is concluding that DART has made a potentially significant contribution to the entire transit industry. Below is a Detailed Analysis of the DART Optimal Operator Report.

\section{Detailed Analysis of DART Optimal Operator Report}

Total Blocks of Work - Full Time and Part Time

The first line item in the instrument establishes the number of FTE blocks of work for fulltime and part-time shifts. This number is developed by Service Planning at the conclusion of each bid and remains constant until the next bid. Individual values are included for each month over a twelve month period and there is a projection for the month that is 60 days beyond the final month of data values. With payroll, data for any given month is usually not available until the end of the following month. In the case of total blocks of work, there were 1,008 full-time and 26 part-time blocks through February of 2007; however, a new bid in April would create 1,016 full time blocks and 26 part-time blocks.

\section{Extraboard Requirement}

Over time, DART included, added, and deleted various categories of absences in order to determine the required extraboard. The final instrument tracks seven categories of leave which are discussed in detail below. 


\section{$\underline{\text { Unscheduled Absences }}$}

Unscheduled absences are a derived value whereupon the total hours of unscheduled absences for the month are divided by 8 hours (defined as FTE) and then divided by 160 hours (defined as one month) to determine the total FTE extraboard operators needed due to runs not covered in this absence category. Based on a rolling average, a total of 92 FTE bus operators are needed to cover unscheduled absences.

\section{FMLA (all categories - using VAC/SICK/LWP)}

This category includes the FTE bus operator positions needed for employees on scheduled leave due to the Family Medical Leave Act. The derivation is the same for unscheduled absences. Based on a rolling average for DART, 11 FTE positions are needed on a monthly basis to cover FMLA leave.

\section{Alternative Duty}

This category includes the FTE bus operator positions needed for employees on alternative duty due to injury/workman's comp or other duties as defined by the DART organization. Based on a rolling average, 17 FTE positions are needed on a monthly basis to cover Alternative Duty leave.

\section{Scheduled Absences}

This category included absences due to vacation, pre-planned sick leave, jury duty or other types of absences defined by DART. FTE's are calculated by the payroll system and a rolling average shows that 108 FTE positions are needed on a monthly basis to cover scheduled leave.

\section{Non-operating Function}

This category includes FTE positions performing work that is not calculated in the Total FTE Blocks of Work needed to run scheduled service (e.g. special services or other functions defined by DART). The rolling average shows that a total of 5 FTE positions are needed to cover non-operating functions. 


\section{Operator Training}

This category includes absences due to full-time or part-time operators participating in agency training. The values in this field are derived from the payroll system. The rolling average, based on three consecutive months prior to month of projection, shows that 10 FTE's are needed on a monthly basis to cover operators in training.

\section{$\underline{\text { Union Business }}$}

This category includes absences due to authorized union business. The values in this field are derived from the payroll system. The rolling average shows that 3 FTE positions are needed on a monthly basis to cover absences due to union business.

\section{$\underline{\text { Total Extraboard Requirement }}$}

The total extraboard requirement is a sum of all seven categories of leave that are tracked by DART. Over a 12 month period, the range of extraboard operator needs was as low as 216 and as high as 285 FTE positions. The trend for the three month period is 249 FTE's while the average is 246 FTE positions.

\section{Percentage of Extraboard}

According to DART, the calculation for the percentage of extraboard is the extraboard requirement plus turnover divided by the FT optimal bus forecast for needed positions. The rolling trend for the three month period is 20.3 percent while the rolling average for the three month period is 20.1 percent of total optimal bus forecast operators.

\section{Turnover}

\section{$\underline{\text { Terminations/Promotions/Transfers to and from Train }}$}

In terms of the optimal bus forecast, turnover is calculated as the sum of terminations, promotions and transfers to train subtracting the transfers from train. The percentage of turnover is calculated as total turnover divided by current staffing. 


\section{Optimal Bus Forecast - Full-Time and Part-Time Operators}

The optimal bus forecast provides the synthesized value for the entire instrument by displaying the total number of full-time and part-time operators by taking into account the total number of blocks of work, the required size of the extraboard, and the rate of turnover. The optimal bus forecast is the number that provides DART the measurement of whether recruiting levels are keeping pace with the total required workforce. As of the March 2007 Optimal Operator Report, the trend is that there are 1,042 blocks of work and the optimal forecast from a trend perspective is 1,300 . This means that the optimal forecast of operators is 125 percent of total blocks of work, with that 25 percent including the extraboard sizing and rates of attrition.

Current Staffing - Full-Time and Part-Time

Current staffing displays total operators by month. As of the March 2007 Optimal Operator Report, the trend is 1,243 full-time and part-time operators, which is 95.6 percent of the optimal bus forecast.

\section{Students in Training -- Graduating Next Month}

DART staff indicated that all recruitment training classes are scheduled for the year such that months of graduation are known in advance. Therefore, each month is populated with either a "0" value or expected graduations for next month based on the pre-determined graduation schedule. This data field does not carry a trend or average for projections since they are actual values.

\section{Required Recruitment Level - Full and Part Time}

This field displays for DART the degree to which the optimal bus forecast is being met by required recruiting levels. The values by month fluctuate significantly with a high of 110 in June 2006 and a low of 24 in September 2006, based on graduations from training. However, as of the March 2007 Optimal Operator Report, the report shows that from a trend perspective DART needs to recruit an additional 57 operators in order to meet the optimal bus forecast. This forecast shows that DART is experiencing difficulties in recruiting operators which is consistent with anecdotal evidence cited around the country. 


\section{APPLICATION OF DART OPTIMAL OPERTOR REPORT TO FLORIDA TRANSIT AGENCY}

In order to validate the DART Optimal Operator Report and establish viability of this instrument for all transit agencies and for the industry, NCTR researchers selected a Florida transit system to collect data and populate the report for that agency's use. VOTRAN is the transit system selected for the following reasons:

- VOTRAN is the largest of the small transit agencies.

- VOTRAN has the highest number and percentage of total extraboard operators for its peer group in the survey.

Therefore, applying the DART instrument to VOTRAN will give researchers and VOTRAN a means by which to compare their actual extraboard staffing levels with the suggested staffing levels indicated by the DART instrument.

VOTRAN completed the DART instrument using data from their payroll system and other operations reports. Staff indicated it took about eight to ten hours to compile the data and complete the instrument. Although VOTRAN reported that they had 20 full time designated extraboard operators in the survey, the agency now indicates that it has 14 designated extraboard operators. As compared to DART, VOTRAN has very low turnover rates. The instrument indicates that the total need is 12 extraboard operators. However, VOTRAN's optimal staffing level based on the instrument is 94 total operators and the agency currently employs 101 operators. Therefore, based on DART's instrument the agency actually has a surplus of bus operator employees and therefore a negative number in the Required Recruitment Level field, indicating that there is no recruiting shortage. VOTRAN did indicate that the agency would continue using the Optimal Operator Report. 


\section{Table 3-1}

JUNE 2007 VOTRAN OPTIMAL OPERATOR REPORT - THREE MONTH ROLLING AVERAGE






\section{GUIDE FOR ALL TRANSIT AGENCIES}

Table 3-2 below provides a detailed methodology for each absence category to show data needs and formulas to calculate the model Optimal Bus Forecast that was developed by the Dallas Area Rapid Transit (DART) district. 
Table 3-2

Guide for Calculating Optimal Bus Forecast (DART)

\begin{tabular}{|c|c|c|}
\hline $\begin{array}{l}\text { TOTAL FTE BLOCKS OF WORK - FULL } \\
\text { TIME }\end{array}$ & & Calculation of total FT blocks - weekday \\
\hline $\begin{array}{l}\text { TOTAL FTE BLOCKS OF WORK - PART } \\
\text { TIME }\end{array}$ & & Calculation of total PT blocks - weekday \\
\hline Extraboard Requirement* & $\begin{array}{l}\text { Unscheduled Absences } \\
\text { FMLA (all categories - using } \\
\text { VAC/Sick/LWP) } \\
\text { Alternative Duty } \\
\text { Scheduled Absences } \\
\text { Non-operating Function } \\
\text { Operator Training } \\
\text { Union Business }\end{array}$ & $\begin{array}{l}\text { For each of the absence categories, take the total } \\
\text { person absences and multiply by } 8, \text { then divide } \\
\text { by } 160 \text {. In months where there are three pay } \\
\text { periods, divide by } 240\end{array}$ \\
\hline TOTAL EXTRABOARD REQUIREMENT & & $\begin{array}{l}\text { Sum of all absence categories to create FTE } \\
\text { extraboard requirement per month }\end{array}$ \\
\hline PERCENTAGE OF EXTRABOARD & & $\begin{array}{l}\text { Calculation of \% for the extraboard is the } \\
\text { extraboard requirements + total turnover / FT } \\
\text { optimal bus forecast. }\end{array}$ \\
\hline $\begin{array}{c}\text { Turnover - \# as of end of } \mathbf{m t h}^{*} \\
\text { Terminations } \\
\end{array}$ & & $\begin{array}{l}\text { Actual number of terminations in a calendar } \\
\text { month }\end{array}$ \\
\hline Promotions & & Actual number of promotions in a calendar month \\
\hline Transfers to train & & \begin{tabular}{|l|} 
For non-rail systems, this includes the actual \\
transfers to any other department in the agency
\end{tabular} \\
\hline Total Turnover & & Sum of terminations, promotions and transfers \\
\hline Transfers from Train * & & $\begin{array}{l}\text { Transfers from any other position to bus operator } \\
\text { is a net gain }\end{array}$ \\
\hline PERCENTAGE OF TURNOVER & & $\begin{array}{l}\text { The turnover \% is determined by dividing the } \\
\text { total turnover/current staffing }\end{array}$ \\
\hline Optimal Bus Forecast* & & \\
\hline & Full-Time Bus Operators & $\begin{array}{l}\text { Total blocks of work + total extraboard + total } \\
\text { turnover FT category }\end{array}$ \\
\hline & Part-Time Bus Operators & $\begin{array}{l}\text { Total blocks of work }+ \text { total extraboard + total } \\
\text { turnover PT category }\end{array}$ \\
\hline TOTAL OPTIMAL BUS FORECAST & & Sum of FT + PT Operators \\
\hline Current Staffing - \# as of end of qtr. & & \\
\hline & Full-Time & $\begin{array}{l}\text { Total FT currently employed by month + students } \\
\text { in training }\end{array}$ \\
\hline & Part-Time & $\begin{array}{l}\text { Total PT currently employed by month + students } \\
\text { in training }\end{array}$ \\
\hline TOTAL CURRENT STAFFING & & Sum of FT + PT Operators \\
\hline Students in training, graduating next Month & & $\begin{array}{l}\text { Assumes that training classes and graduations } \\
\text { are pre-established annually }\end{array}$ \\
\hline $\begin{array}{l}\text { REQUIRED RECRUITMENT LEVEL - FULL } \\
\text { TIME }\end{array}$ & & Target number of student operators needed FT \\
\hline $\begin{array}{l}\text { REQUIRED RECRUITMENT LEVEL - PART } \\
\text { TIME }\end{array}$ & & Target number of student operators needed PT \\
\hline Average Formula & & $\begin{array}{l}\text { Sum of data from each category divided by } 12 \\
\text { months }\end{array}$ \\
\hline Trend Formula & $\begin{array}{l}\text { Uses related known values to predict } \\
\text { unknown (future) values }\end{array}$ & Formula established in Excel \\
\hline
\end{tabular}




\section{SECTION FOUR \\ SURVEY Of States \\ Hours of Service (HOS) For Drivers Carrying Passengers}

The Florida Department of Transportation requested that NCTR conduct a survey of the states relative to CFR 49, Part 395, the Federal Motor Carrier Safety Administration (FMCSA) Hours of Service (HOS) for Drivers Carrying Passengers. This regulation provides a standard for maximum hours of work, minimum hours off duty, and the maximum period (spread) of work time, both driving and not driving. Section 395.5 states the following:

\section{$\S 395.5$ Maximum driving time for passenger-carrying vehicles.}

Subject to the exceptions and exemptions in $\underline{\S 95.1}$ :

(a) No motor carrier shall permit or require any driver used by it to drive a passengercarrying commercial motor vehicle, nor shall any such driver drive a passenger-carrying commercial motor vehicle:

(a)(1) More than 10 hours following 8 consecutive hours off duty; or

(a)(2) For any period after having been on duty 15 hours following 8 consecutive hours off duty.

(b) No motor carrier shall permit or require a driver of a passenger-carrying commercial motor vehicle to drive, nor shall any driver drive a passenger-carrying commercial motor vehicle, regardless of the number of motor carriers using the driver's services, for any period after-

(b)(1) Having been on duty 60 hours in any 7 consecutive days if the employing motor carrier does not operate commercial motor vehicles every day of the week; or

(b)(2) Having been on duty 70 hours in any period of 8 consecutive days if the employing motor carrier operates commercial motor vehicles every day of the week.

[68 FR 22516, Apr. 28, 2003; 70 FR 50073, Aug. 25, 2005] 
Section 395.1(a)(2) states that, "The exceptions from Federal requirements found in paratransits $(\mathrm{l})$ through $(\mathrm{n})$ do not preempt State laws and regulations governing the safe operation of motor vehicles." In other words, States have the option to adopt the federal standard, create exceptions to the standard, or provide more stringent standards than the federal standard.

CUTR worked with the American Association of State Highway and Transportation Officials (AASHTO) Standing Committee on Public Transportation (SCOPT) to develop a mailing list and survey to determine how other states and transit systems monitor and control total days worked, on-duty hours, driving hours and time reporting on and off duty each day. To date, CUTR has garnered data on 39 states based on the survey and independent research of state web sites. Since this particular regulation is found in laws related to motor carrier safety and not transit (FTA) laws and regulations, sometimes States have different regulatory agencies that are not related to the State DOT in charge of hours of service requirements. The eleven states where there is currently no data to report include:

- Arizona

- Hawaii

- lowa

- Mississippi

- Nevada

- New Jersey

- Rhode Island

- South Dakota

- Tennessee

- Vermont

- Virginia

Of the 39 states where data is available, 30 states incorporate the federal standard of CFR 49 Part 395.5. The remaining nine states that have exceptions and/or different standards are as follows:

- Arkansas

- Colorado

- Florida 
- Massachusetts

- Missouri

- Nebraska

- New York

- South Carolina

- Washington

Table 4-1 below displays the language of exception for each of these nine states and the source from which the regulations were found. It should be noted that many states have exceptions to the federal regulations in specific industries such as agriculture, utilities, emergency vehicles, etc. Since these industries were not relevant to FDOT's request, CUTR's efforts were focused on the states' requirements for transit bus drivers to the extent the information was available. 
Table 4-1

\section{States with Exceptions to the Federal Standard Hours of Service (HOS) for Drivers Carrying Passengers}

\begin{tabular}{|c|c|c|}
\hline State & Hours of Service Regulation & Source or Contact \\
\hline Arkansas & $\begin{array}{l}\text { It shall be unlawful for any companies, firms, or corporations, or officers of courts or individuals owning, } \\
\text { operating, leasing, or subleasing any lines using vehicles propelled by any form of energy on the highways } \\
\text { of Arkansas for the purpose of transporting passengers, freight, mail, express, or any commodity to keep } \\
\text { their drivers on duty more than fifteen (15) consecutive hours. At the expiration of fifteen (15) hours of duty, } \\
\text { the driver must have at least eight (8) hours of rest. }\end{array}$ & http://www.arkansas.gov/labor/code.php?code=23-13-101 \\
\hline Colorado & $\begin{array}{l}\text { A passenger-carrying commercial motor vehicle driver has at least } 8 \text { consecutive hours off duty separating } \\
\text { each } 12 \text { hours on duty, and does not exceed } 10 \text { hours maximum driving time following } 8 \text { consecutive hours } \\
\text { off duty. }\end{array}$ & http://csp.state.co.us/downloads/compliancereview.pdf \\
\hline Florida & $\begin{array}{l}\text { (3) The driver of a bus shall not be permitted or required to drive more than } 12 \text { hours in any one } 24 \text {-hour } \\
\text { period, or drive after having been on duty for } 16 \text { hours in any one } 24 \text {-hour period, or drive more than } 70 \\
\text { hours in any period of seven consecutive calendar days. } \\
\text { (a) A driver who has reached the maximum } 12 \text { driving hours or } 16 \text { hours on duty time shall be required to } \\
\text { have a minimum of eight consecutive hours off duty time within any one } 24 \text {-hour period. } \\
\text { (b) A driver's work period shall begin from the time a driver first reports for duty for his or her employer. } \\
\text { (4) A driver may be permitted to drive for more than the regulated hours if the hours are necessitated by } \\
\text { adverse conditions resulting from weather, road or traffic, or emergencies resulting from an accident, } \\
\text { medical reasons or disaster. } \\
\text { (5) The driver of a bus may be permitted to exceed his or her regulated hours in order to reach a regularly } \\
\text { established relief point, provided the additional driving time does not exceed one hour. }\end{array}$ & Chapter 14-90, Florida Administrative Code \\
\hline Massachusetts & $\begin{array}{l}\text { Hours of Service. No owner of a motor bus shall cause or allow any driver to drive a motor bus for more than } \\
\text { ten hours in any period of } 24 \text { consecutive hours, unless such driver be afforded eight consecutive hours of } \\
\text { rest immediately following the ten hours aggregate drive. }\end{array}$ & http://www.mass.gov/Eoca/docs/dte/cmr/220cmr155.pdf \\
\hline Missouri & $\begin{array}{l}\text { RSMo } 307.400,390.063,622.550,390.201 \text { are the Missouri statutes that bring FMCSR applicability to } \\
\text { carriers within Missouri. If the carrier is subject to the FMCSR's, then as a passenger carrier they are limited } \\
\text { by the Hours-of-Service (HOS) rules in Part } 395 \text { to } 10 \text { hours of driving, a } 15 \text { hour total work-day, and must } \\
\text { have } 8 \text { hours off-duty in-between work days. Federal, state, and local government operations (non- } \\
\text { contracted services run/staffed by the government) are exempt from the most of FMCSR's including HOS } \\
\text { per Part } 390.2 \text {. So if exempt, then only their policies and common sense would limit their work and driving } \\
\text { times. }\end{array}$ & $\begin{array}{l}\text { Darrell E Chute, Senior Motor Carrier Specialist, } \\
\text { MODOT/Motor Carrier Services Division/Transportation Safety } \\
\text { \& Compliance Office 537-751-1853 }\end{array}$ \\
\hline Nebraska & 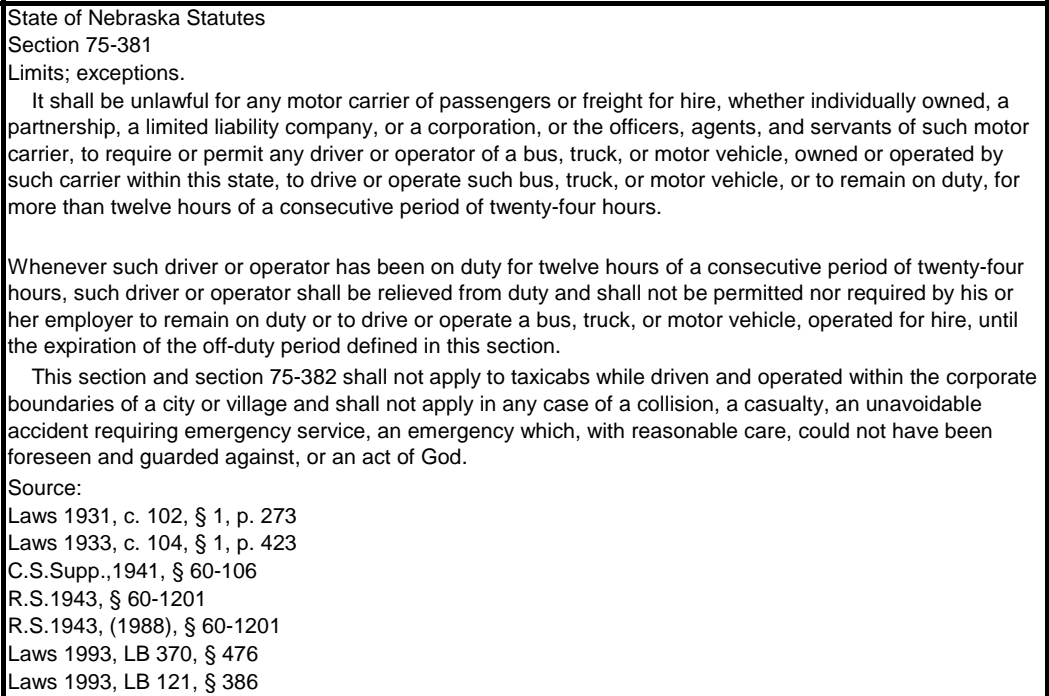 & http://srvwww.unicam.state.ne.us/Statutes2005.html \\
\hline
\end{tabular}


Table 4-1 (Continued)

\section{States with Exceptions to the Federal Standard Hours of Service (HOS) for Drivers Carrying Passengers}

\begin{tabular}{|c|c|c|}
\hline State & Hours of Service Regulation & Source or Contact \\
\hline New York & 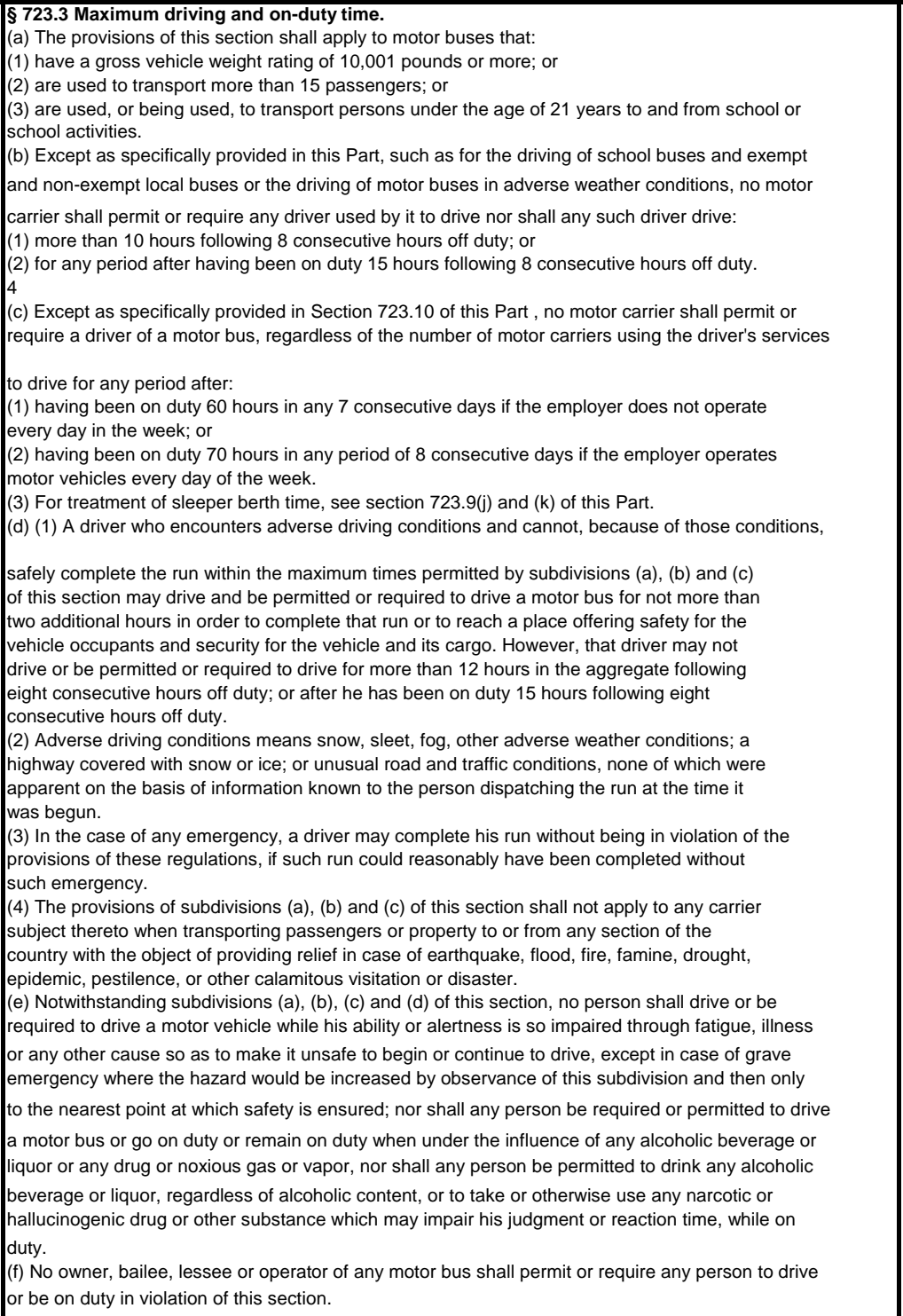 & http://www.dot.state.ny.us/ts/files/busregs.pdf \\
\hline South Carolina & $\begin{array}{l}\text { South Carolina DOT would like to inform you that we have no state regulations or statutes regarding bus } \\
\text { operator's "on-duty" and/or "drive" hours for public transportation operators. Instead, our operators follow } \\
\text { the Federal Motor Carrier Safety Regulations; specifically, Part } 395.5 \text { "Maximum driving time for passenger- } \\
\text { carrying vehicles". Since our state's public transportation operators are all publicly-operated systems, work } \\
\text { rules ARE somewhat different than they would be for privately-operated services. Operators generally use } \\
\text { "bid runs" which follow the "10 on/8 off" rule (as per 49CFR Part 395.5). Of course, being publicly operated } \\
\text { systems, operators here have a great deal of flexibility regarding scheduling since all drivers are "local" } \\
\text { drivers; that is, they never leave a relatively small area and their "runs" are short as compared to the long- } \\
\text { distance, over-the-road private system driver. This makes it easy to "recall" them should they be needed to } \\
\text { work "extra hours" which would be hours over the } 10 \text { duty hours they are scheduled for. When they do this, } \\
\text { drivers have to be paid for overtime and still be given } 8 \text { hours off for rest or the bus operator may risk being } c\end{array}$ & Jim Frierson, 803-737-3601 or e-mail at friersonjm@scdot.org \\
\hline Washington & $\begin{array}{l}\text { No person, agent, officer, manager, or superintendent or receiver of any corporation or owner of streetcars } \\
\text { shall require his, her, or its gripmen, motormen, drivers, or conductors to work more than ten hours in any } \\
\text { twenty-four hours. All other references in State law refer back to the regulations adopted by USDOT. }\end{array}$ & Savary, Barb [SavaryB@wsdot.wa.gov] \\
\hline
\end{tabular}




\section{Conclusion}

In June 2007, the results of this research were presented to the Florida Operations Managers as part of the Florida Operations Network session of the Florida Public Transit Association's mid-year conference. The Operations managers were enthusiastic about the DART instrument for optimal operator forecasting but requested further testing to ensure that the instrument can be applied to any transit agency. The VOTRAN case study suggests that the instrument can be universally applied. The operations managers also cited issues with bus operator recruitment and retention and many indicated there is a shortage of available work force. Other factors that influence workforce needs in Florida include the following:

- Absenteeism is more complex than in the past with the FMLA resulting in unpredictable and longer leaves of absence.

- Operator salaries in many Florida markets are not keeping pace with rising cost of living.

- In a hot job market, transit employees can either find higher paying jobs in the trucking industry or other sectors of the economy.

- Recruiting shortages in many areas are driving the cost of overtime in order to meet scheduled service. Operations managers are receiving complaints from upper management about the rising costs of overtime.

- When staffing levels reach significantly low levels, overtime is forced on operators which can contribute to burnout, fatigue, and increased levels of unscheduled absenteeism.

- Using actual numbers of extraboard operators for any one system is not necessarily going to reflect the true need based on total staffing requirements. This is where the DART instrument is especially helpful because it displays conclusively the levels of recruitment needed in order to maintain optimal staffing levels.

- Future research should include strategies for recruitment, salaries, and incentives to achieve employee retention and absenteeism reduction. 


\section{Bibliography}

American Public Transportation Association. (2003). Organizational website. Retrieved May 2007, from http://www.apta.com/services/safety/glossary.cfm

Koutsopoulos, Haris N. (1990). Scheduling of Extraboard Operators in Transit Systems. Transportation Science. Volume 24, No. 2: 87-104.

Long, Lyn and James L. Perry. (1984). Extraboard Scheduling, Workers' Compensation and Operator Stress in Public Transit: Research Results and Managerial Implications. The Institute of Transportation Studies at the University of California, Irvine. UCI-ITS-WP-84-4.

MacDorman, L.C. (1985). Extraboard Management: Procedures and Tools. National Cooperative Transit Research and Development Program, Synthesis of Transit Practice, Report No. 5.

Transportation Research Record 1002: Labor and Manpower Management Issues, TRB, 1984.

Wilson, Nigel H.M. (2003). Public Transportation Service and Operations Planning: Workforce Planning. 


\section{Appendix A \\ Transit Agency Survey Extraboard Management}


Name:

Phone number:

Transit system:

Email address:

\begin{tabular}{|c|c|c|c|}
\hline \multicolumn{4}{|l|}{ Basic System Information } \\
\hline & Total & $\begin{array}{l}\text { Full } \\
\text { Time }\end{array}$ & Part Time \\
\hline \multicolumn{4}{|l|}{ What is your total number of bus operator positions (filled + unfilled)? } \\
\hline & Weekday & Saturday & Sunday \\
\hline \multicolumn{4}{|l|}{$\begin{array}{l}\text { What is your total number of daily revenue hours for the most recent } \\
\text { bid? }\end{array}$} \\
\hline & Weekday & Saturday & Sunday \\
\hline \multicolumn{4}{|l|}{$\begin{array}{l}\text { For your most recent bid, how many of the following types of shifts do } \\
\text { you have? }\end{array}$} \\
\hline \multirow{5}{*}{\multicolumn{4}{|c|}{$\begin{array}{r}8 \text { hour straights } \\
10 \text { hour straights } \\
\text { Splits } \\
\text { Open pieces of work } \\
\text { Other (please describe) }\end{array}$}} \\
\hline & & & \\
\hline & & & \\
\hline & & & \\
\hline & & & \\
\hline & Full Time & $\begin{array}{l}\text { Part } \\
\text { Time }\end{array}$ & \\
\hline $\begin{array}{l}\text { Of your total number of bus operators, how many are designated } \\
\text { extraboard? }\end{array}$ & & & \\
\hline
\end{tabular}

\begin{tabular}{|l|c|c|}
\hline Labor Agreement & \multicolumn{2}{|c|}{ No } \\
\hline $\begin{array}{l}\text { Do management and labor have a collective bargaining agreement at } \\
\text { your agency? }\end{array}$ & $\square$ & \\
\hline $\begin{array}{l}\text { Does the agreement address limitations in the number or percentage of } \\
\text { full time and part time employees? }\end{array}$ & $\square$ & $\square$ \\
\hline $\begin{array}{l}\text { Does the agreement address policies, rules, deployments, seniority, } \\
\text { overtime and/or other provisions related to extraboard management? }\end{array}$ & $\square$ & $\square$ \\
\hline
\end{tabular}

Please summarize below or provide the section of the labor agreement via e-mail (if possible) that addresses extraboard.

\begin{tabular}{|l|l|l|}
\hline Absenteeism and Attrition & \multicolumn{2}{|l|}{ No } \\
\hline $\begin{array}{l}\text { The following questions pertain to any individual bid and/or mark- } \\
\text { up period }\end{array}$ & Yes & \\
\hline $\begin{array}{l}\text { Do you have an established percentage or number for attrition of } \\
\text { employees through resignation, termination and retirement? }\end{array}$ & $\square$ \\
\hline If yes, what percentage or number do you use for attrition? & \\
\hline $\begin{array}{l}\text { Do you have an established percentage or number for scheduled leave } \\
\text { to include vacation, training, union business? }\end{array}$ & $\square$ \\
\hline If so, what percentage or number do you use for scheduled leave? & \\
\hline $\begin{array}{l}\text { Do you have an established percentage or number for unscheduled } \\
\text { leave to include sick, jury duty, administrative leave? }\end{array}$ & $\square$ & $\square$ \\
\hline If so, what percentage or number do you use for unscheduled leave? & $\square$ \\
\hline
\end{tabular}




\begin{tabular}{|l|c|}
\hline Scheduling & \multicolumn{2}{|l|}{ Yes } & No \\
\hline $\begin{array}{l}\text { Do you use automated scheduling software for master schedules, } \\
\text { runcuts, and/or interlines? }\end{array}$ & $\square$ \\
\hline $\begin{array}{l}\text { If yes, does your scheduling software calculate number of extraboard } \\
\text { operators required? }\end{array}$ & $\square$ \\
\hline $\begin{array}{l}\text { If yes, has it been your experience that your software accurately } \\
\text { calculates the true number of extraboard employees needed? }\end{array}$ & $\square$ \\
\hline $\begin{array}{l}\text { Do you have a number or percentage of extraboard operators needed } \\
\text { for each bid? If yes, what is that percentage? }\end{array}$ & $\square$ \\
\hline
\end{tabular}

\section{Operations}

We would like information regarding the type of work that is available for extraboard operators.

The order that extraboard operators receive (or pick) their work assignment generally remains the same.

The order that extraboard operators receive (or pick) their work assignment is based on a rotating system.

If yes to previous question, how often does the order change? Daily Weekly By pick or mark-up period.

Work assignments which are picked by extraboard operators include: Assignments held down for more than one day. Known open work posted for the next day. Unanticipated open work (e.g. miss outs) Other (e.g. special work assignments) Not applicable

Work assignments which are assigned to extraboard operators by the dispatchers with operator's option to pass include: Assignments held down for more than one day Known open work posted for the next day. Unanticipated open work (e.g. miss outs) Other (e.g. special work assignments) Not applicable

\section{Operations - Continued}

Work assignments which are assigned to extraboard operators by the dispatchers without operator's option to pass include:

Assignments held down for more than one day.

Known open work posted for the next day.

Unanticipated open work (e.g. miss outs)

Other (e.g. special work assignments)

Not applicable 


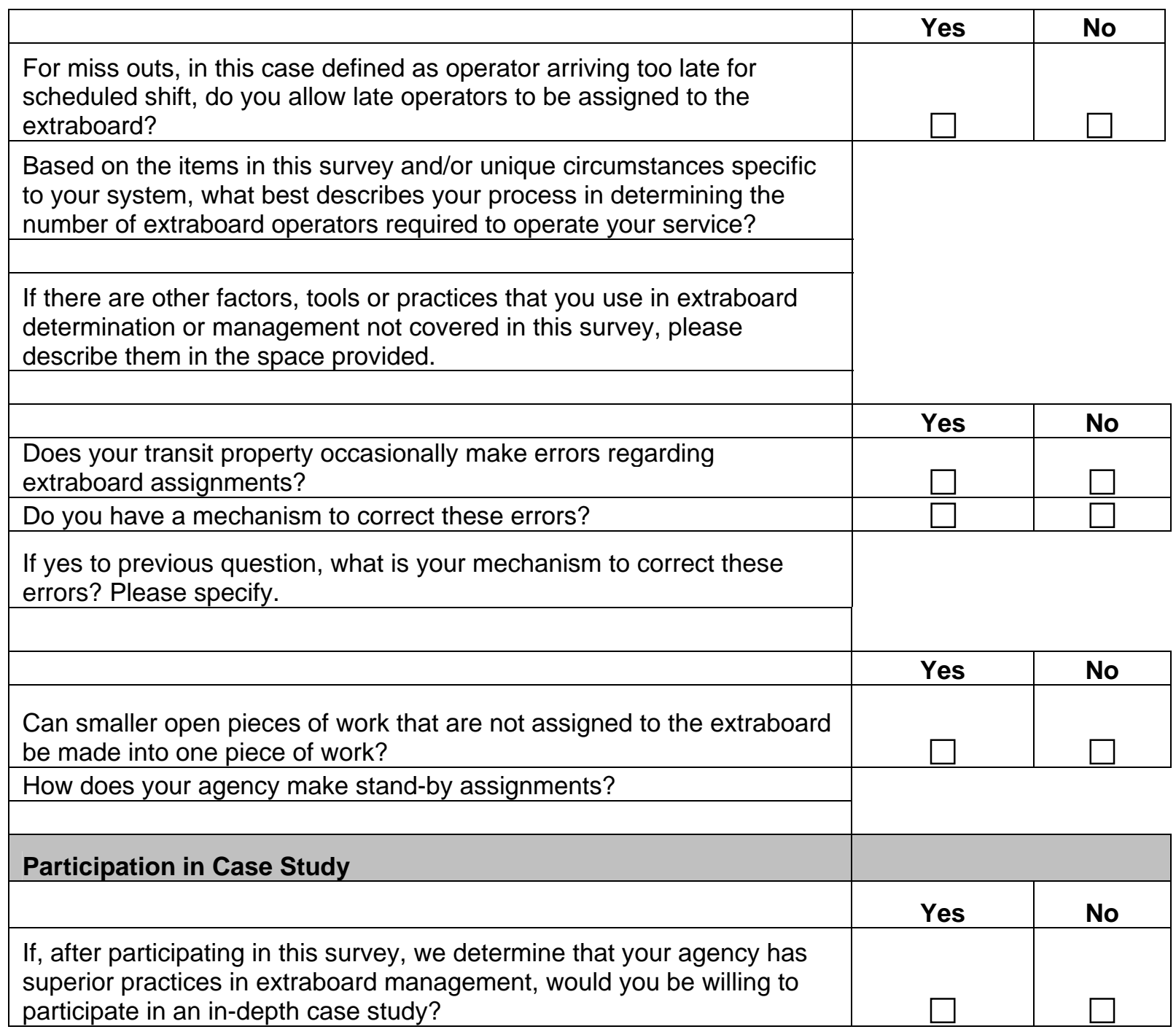

